\title{
Urotropin azelate: a rather unwilling co-crystal
}

\section{Michel Bonin, T. Richard Welberry, Marc Hostettler, Manuel Gardon, Henrik Birkedal, Gervais Chapuis, Pedro Möckli, Craig A. Ogle and Kurt J. Schenk}


Acta Crystallographica Section B

Structural

Science

ISSN 0108-7681

\section{Michel Bonin, ${ }^{\mathrm{a}}$ T. Richard Welberry, ${ }^{b}$ Marc Hostettler, ${ }^{\text {a }}$ Manuel Gardon, ${ }^{a}$ Henrik Birkedal, ${ }^{a}$ Gervais Chapuis, ${ }^{\text {a }}$ Pedro Möckli, ${ }^{\mathrm{c}}$ Craig A. Ogle ${ }^{\mathrm{d}}$ and Kurt J. Schenk ${ }^{\mathrm{a} *}$}

anstitut de Cristallographie, Université de Lausanne, BSP Dorigny, CH-1015 Lausanne, Switzerland, ${ }^{\mathbf{b}}$ Research School of Chemistry, Australian National University, Canberra, ACT 0200, Australia, 'Department for Ceramics, Swiss Federal Technology Institute, $\mathrm{CH}-1015$ Lausanne, Switzerland, and ${ }^{\mathbf{d}}$ Chemistry Department, University of North Carolina Charlotte, 9210 University City Blvd, Charlotte, NC 28223-0001, USA

Correspondence e-mail: kurt.schenk@ic.unil.ch

\section{Urotropin azelate: a rather unwilling co-crystal}

Urotropin (U) and azelaic acid (AA) form 1:1 co-crystals (UA) that give rise to a rather complex diffraction pattern, the main features of which are diffuse rods and bands in addition to the Bragg reflections. UA is characterized by solvent inclusions, parasite phases, and high vacancy and dislocation densities. These defects compounded with the pronounced tendency of $U$ to escape from the crystal edifice lead to at least seven exotic phase transitions (many of which barely manifest themselves in a differential scanning calorimetry trace). These involve different incommensurate phases and a peritectoid reaction in the recrystallization regime $\left(T_{h}>0.6\right)$. The system may be understood as an OD (order-disorder) structure based on a layer with layer group $P(c) c 2$ and cell $a_{o} \simeq 4.7, b \simeq 26.1$ and $c \simeq 14.4 \AA$. At $338 \mathrm{~K}$ the layer stacking is random, but with decreasing temperature the build-up of an orthorhombic MDO (maximal degree of order) structure with cell $\mathbf{a}_{1}=2 \mathbf{a}_{o}$, $\mathbf{b}_{1}=\mathbf{b}, \mathbf{c}_{1}=\mathbf{c}$ and space group Pcc2 is begun (at $\left.\sim 301 \mathrm{~K}\right)$. The superposition structure of the OD system at $T=286$ (1) K with space group $B m m b$ and cell $\widehat{\mathbf{a}}=2 \mathbf{a}_{o}, \widehat{\mathbf{b}}=\mathbf{b}$ and $\widehat{\mathbf{c}}=\mathbf{c} / 2$ owes its cohesion to van der Waals interactions between the AA chains and to three types of hydrogen bonds of varied strength between $\mathrm{U}-\mathrm{U}$ and $\mathrm{U}-\mathrm{AA}$. Before reaching completion, this MDO structure is transformed, at $282 \mathrm{~K}$, into a monoclinic one with cell $\mathbf{a}_{m}=-\mathbf{a}_{o}+\mathbf{c} / 4, \mathbf{b}_{m}=\mathbf{b}$, $\mathbf{c}_{m}=-2\left(\mathbf{a}_{o}+c / 2\right)$, space group $P 2_{1} / c$, spontaneous deformation $\sim 2^{\circ}$, and ferroelastic domains. This transformation is achieved in two steps: first a furtive triggering transition, which is not yet fully understood, and second an improper ferroelastic transition. At $\sim 233 \mathrm{~K}$, the system reaches its ground state (cell $\mathbf{a}_{M}=\mathbf{a}_{m}, \mathbf{b}_{M}=\mathbf{b}, \mathbf{c}_{M}=\mathbf{c}_{m}$ and space group $\left.P 2_{1} / c\right)$ via an irreversible transition. The phase transitions below $338 \mathrm{~K}$ are described by a model based on the interaction of two thermally activated slip systems. The OD structure is described in terms of a three-dimensional Monte Carlo model that involves first- and second-neighbour interactions along the $a$ axis and first-neighbour interactions along the $b$ and $c$ axes. This model includes random shifts of the chains along their axes and satisfactorily accounts for most features that are seen in the observed diffraction pattern.

\section{Introduction}

Urotropin (U), besides its obvious medical importance (ov $\rho \mathrm{v} v$ - $\tau \rho \epsilon \pi \epsilon \iota \nu)$, distinguishes itself by forming almost perfect single crystals with very low dislocation density $\left(<1 \mathrm{~mm}^{-2}\right.$; DiPersio \& Escaig, 1972) and a mosaic spread half-width of 7" (Kampermann et al., 1995). It is a much studied classic capable of the most surprising applications. On the one hand the quasispherical molecule does not have a plastic phase but forms a
Received 16 July 2002 Accepted 26 November 2002

Dieter Schwarzenbach zu seinem Ruhestand gewidmet. 'Deine umfassende kristallographische Kultur wird uns immer Vorbild sein.'
C 2003 International Union of Crystallography Printed in Great Britain - all rights reserved 
very compact structure (packing coefficient 0.74 ) that persists from 15 to $548 \mathrm{~K}$, at which temperature sublimation occurs (Klipping \& Stranski, 1958). U owes this behaviour to the rather weak $(2.866 \AA) \mathrm{C}-\mathrm{H} \cdots \mathrm{N}$ hydrogen bonds that hold the molecules together. The molecules themselves may be treated to a very good approximation as rigid bodies (libration is $5^{\circ}$ at $200 \mathrm{~K}$ and $2^{\circ}$ at $5 \mathrm{~K}$ ), especially below $200 \mathrm{~K}$ (there is only some $\mathrm{CH}_{2}$ vibration present). The intermolecular correlations (Canut \& Amorós, 1958) between these rigid bodies lead to rather strong TDS in inter-Bragg space. On the other hand the situation is compounded by the tendency of $U$ to start subliming at as low as $330 \mathrm{~K}$ (Birkedal, 1996) and, above $468 \mathrm{~K}$, to chemically (Blažević et al., 1979) and thermally

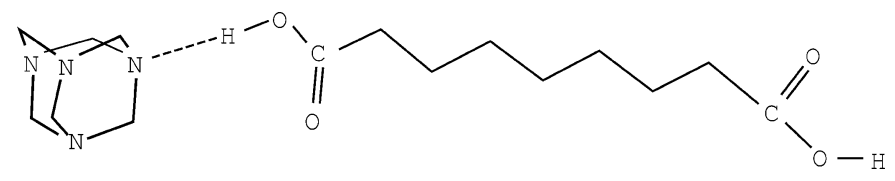

(Klipping \& Stranski, 1958) decompose.

Azelaic acid (AA) crystallizes with packing that owes its cohesion within a layer to van der Waals forces and between layers to $\mathrm{O}-\mathrm{H} \cdots \mathrm{O}$ hydrogen bonds. The stable RT $\beta$ form transforms into the $\alpha$ form at $346 \mathrm{~K}$, but the reverse transition takes place under particular circumstances only (after a long time or under pressure). This mysterious behaviour is especially surprising in view of the similarity in magnitude (to $0.01 \AA$ ) of the hydrogen bonds mentioned above. The reason for the hysteresis must be sought in the chain packing. Here there are several differences. First the packing coefficient for the $\beta$ form is 0.71 while for the $\alpha$ form it is 0.67 . An approximate $p$-cell may be defined for the $\alpha$ form [p-cell is a term used in structures of close-packed long-chain alkanes that relates to the $-\mathrm{CH}_{2}-$ molecular repeat (Abrahamsson et al., 1978)] but not for the $\beta$ form. Finally there are differences in conformation around the $\alpha$-C atoms so that in the $\beta$ form the chains are extended (all trans conformation) while in the $\alpha$ form there is one gauche conformation. Note that both forms develop a different habitus and, even more baffling, that under certain conditions both forms crystallize from the same solvent at the same temperature (Caspari, 1929). In regard to this last point AA must be considered to be one of the earliest reported examples of dimorphism (Dupré La Tour, 1935). It is also interesting to note that AA plays a (not yet understood) role in the inhibition of the activity of 5- $\alpha$-reductase, which converts testosterone into the fatal dihydrotestosterone (Stamatiadis et al., 1988). Finally, mention should be made of the antibacterial, antimycotic and antitumoral properties of AA.

In view of all the fundamental differences between the two molecules it seemed tempting to combine them in a co-crystal and study the manner in which their often contradictory properties expressed themselves in such a new compound. It was considered that the most exotic behaviour might be expected with regard to the structural packing, molecular motion, static disorder, defects, solvent inclusions, and stability and phase transitions. In this contribution, we present results for the room-temperature phase (II) of urotropin azelate (UA) and an overview of its rather complex phase diagram below $338 \mathrm{~K}$ (phases $\alpha$, II, $f$, III and IV), which was studied by means of calorimetry, orthoscopy, X-ray diffraction and Monte Carlo simulations. This investigation highlights the earlier structural study of phase III (Hostettler et al., 1999) as one of many pieces in the complex puzzle of OD phenomena and phase transitions in this system. Throughout the present report a subscript $m$ will stand for phase III, a subscript $M$ will stand for phase IV and quantities without subscript are with respect to phase II.

\section{Experimental}

\subsection{Synthesis}

FLUKA reagent-grade chemicals were used without further purification. Equimolar quantities of the reagents were dissolved in ethanol (U) and water (AA) and made to react by mixing the two solutions. The resulting white precipitate was recrystallized twice from ethanol. Finally the crystals used in this investigation were grown from a solution in acetonitrile. It was found that these crystals contained some of the solvent. When crystallized in this way UA grows as (010) platelets of varying thickness. As a result of the growth conditions a $\{100\}$ pinacoid was occasionally developed, but $\{101\}$ and $\{10 \overline{1}\}$ pinacoids were found to be always present.

\subsection{Gas chromatography}

Experiments were performed on an HP 5890 gas chromatograph (GC) equipped with a 5\% diphenyl-95\% dimethylpolysiloxane capillary column and an FID detector. The UA crystals for this study were grown at ambient temperature from reagent-grade acetonitrile (FLUKA) and were then airdried. A solution of acetonitrile, $5 \mu \mathrm{l}$ in $10 \mathrm{ml}$ of distilled water $(9.29 \mathrm{mM})$, was found to give a signal of 71.7 at a retention time $t_{R}$ of $0.57 \mathrm{~min}$, while a solution of $40 \mathrm{mg}$ of UA in $2 \mathrm{ml}$ of distilled water $(60.24 \mathrm{mM})$ gave a signal of 4.64 at the same $t_{R}$. Distilled water alone gave no signal whatsoever. Taking into account the different molarities, we find that the $\mathrm{UA} \cdot x\left(\mathrm{H}_{3} \mathrm{CCN}\right)$ solution is roughly a hundred times poorer in acetonitrile than the reference sample. The formula for UA must therefore be written as $\mathrm{UA} \cdot 0.01\left(\mathrm{H}_{3} \mathrm{CCN}\right)$. From the crystal and unit-cell dimensions, we estimate that the crystal contains about $10^{16}$ unit cells of which $10^{11}$ lie at the surface. We know that $1 \%$ of the cells contain one acetonitrile, and thus the crystal contains $10^{14}$ molecules of acetonitrile. It is therefore impossible that the whole of the solvent is at the surface only.

\subsection{Differential scanning calorimetry}

At ambient pressure, the thermotropic phase sequence was investigated by differential scanning calorimetry (DSC) using a Mettler DSC30 system. This was calibrated with In, Zn and 
Table 1

Summary of DSC results for UA.

Values given are averages over several measurements.

\begin{tabular}{lll}
\hline Transition & $T(\mathrm{~K})$ & $\Delta H\left(\mathrm{~J} \mathrm{~g}^{-1}\right)$ \\
\hline I $\rightarrow$ Decomposition & 401 & \\
& & \\
I $\rightarrow$ II & 371 & -2.3 \\
II $\rightarrow$ I & 375 & 1.57 \\
& & \\
II $\rightarrow$ III & 282 & 1.65 \\
III $\rightarrow$ II & 283 & -1.74 not passing through III $\rightarrow$ IV \\
II $\rightarrow$ III $\rightarrow$ IV & 282,236 & $1.65,0.50$ \\
IV $\rightarrow$ II & 283 & -2.1 \\
\end{tabular}

$\mathrm{Pb}$ at a rate of $1 \mathrm{~K} \mathrm{~min}^{-1}$, and all measurements were performed at this rate. They were made in cycles in order to investigate hysteresis and reversibility of the phase transitions. Four phases, of which the transition temperatures and corresponding enthalpies are given in Table 1, have been identified. The transition at $371 \mathrm{~K}$ is reversible, but the melting transition at $401 \mathrm{~K}$ is often indicative of decomposition. This is due to a peritectoid reaction that sets in above $\sim 363 \mathrm{~K}$. The reaction is accompanied by an ever increasing weight loss above $\sim 363 \mathrm{~K}$. This loss is detected by thermogravimetry and is shown by GC and NMR to be due to a massive exodus of $U$ from the cocrystal. The I $\leftrightarrow$ II and II $\leftrightarrow$ III transitions are thermodynamically reversible but subject to slight hystereses of 4 and $1.5 \mathrm{~K}$, respectively. On lowering the temperature (Fig. 1) from ambient temperature, two phase transitions are observed: $\mathrm{II} \rightarrow \mathrm{III}$ at $282 \mathrm{~K}$ and III $\rightarrow$ IV at $236 \mathrm{~K}$. However, on raising the temperature from phase IV, the IV $\rightarrow$ III transition is not observed and the crystal transforms directly to phase II at $283 \mathrm{~K}$ (roughly at the same temperature as the II $\rightarrow$ III transition in a cooling run). The enthalpy of the IV $\rightarrow$ II transition corresponds to the sum of the enthalpies of the $\mathrm{II} \rightarrow$ III and III $\rightarrow$ IV transitions. The III $\rightarrow$ II transition occurs at the same temperature as the IV $\rightarrow$ II transition, provided that phase IV has not been reached. This temperature differs only slightly from the temperature of the II $\rightarrow$ III transition $\left(\Delta T_{\text {trans }} \sim 1.5 \mathrm{~K}\right)$.

\subsection{Optical studies}

Orthoscopic studies under crossed Nicols were performed with a WILD M8 Heerbrugg binocular and a LEITZ SM-POL polarizing microscope. Crystals were immersed in perfluoropolyether oil (RS 3000, Riedel-de Haën). The observations indicate that crystals grown at room temperature (phase II) are single-domain states. The habitus of the crystals is tabular and these $\{010\}$ platelets are bounded by $\{100\}$ pinacoids and $\{101\}$ prisms. The ratio of the lengths of the edges [010] and [101] depends on the growth conditions of the specific crystal and varies within a batch. This phase is birefringent. We used $\lambda$ and $\lambda / 4$ plates in order to determine the principal axes of Fletcher's indicatrix in the (010) plane and the fast and slow propagation directions of light. We found these parameters by comparing the birefringence patterns, with and without plates, using Bloss' table of the different orders of interference colour (Bloss, 1990). The main axes of the indicatrix are along the orthorhombic axes and the fast propagation direction of light lies along a (Fig. 2).

On cooling UA crystals in a stream of nitrogen gas, a twostep phenomenon can be observed at the II $\rightarrow$ III transition:

(i) Formation of (401) domain walls. These are associated with orientational domains and propagate through the entire sample.

(ii) Appearance of a fuzzy phase front associated with a transformation of the (401) domains into ferroelastic (100) domains.

This transformation sets in a few seconds after the creation of the (401) domains, but for some crystals the transition

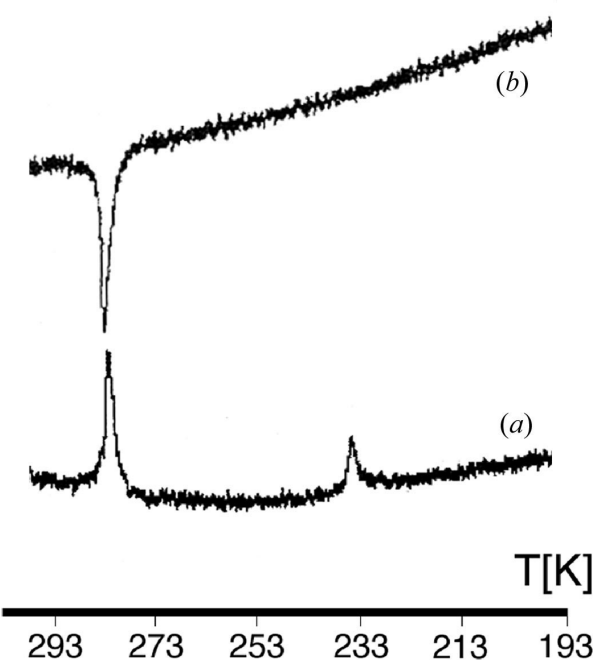

Figure 1

(a) Cooling and (b) heating (in that order) DSC traces at $1 \mathrm{~K} \mathrm{~min}^{-1}$. Note the absence of the IV $\rightarrow$ III transition upon heating.

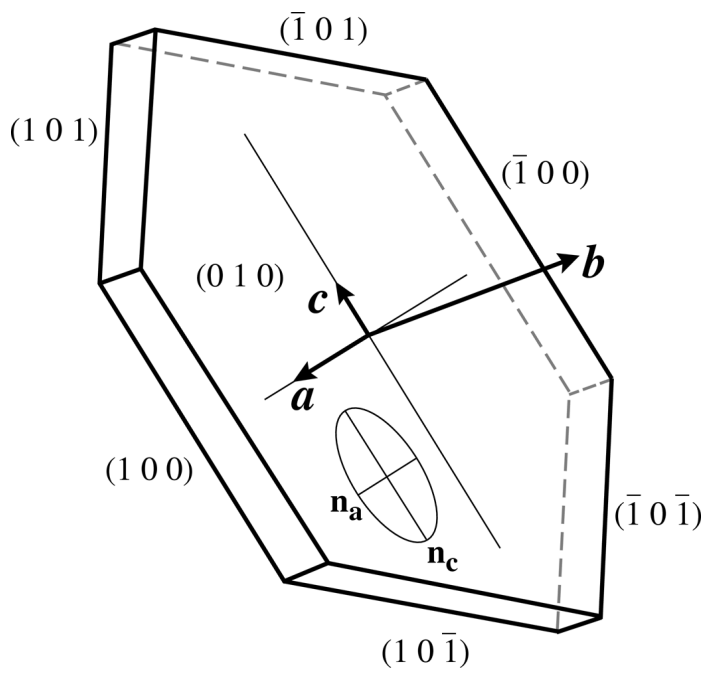

Figure 2

Typical habitus and Fletcher's indicatrix of a UA crystal with respect to the cell of Table 4. 
Table 2

Crystal data of the known solid phases of UA.

\begin{tabular}{|c|c|c|c|}
\hline & Phase II & Phase III & Phase IV \\
\hline Temperature (K) & $286(1)$ & $258(1)$ & $200(1)$ \\
\hline Lattice constants $\left(\AA{ }^{\circ}{ }^{\circ}\right)$ & $\begin{array}{l}\hat{a}=9.416(2), \hat{b}=26.124(5) \\
\quad \hat{c}=7.203(1)\end{array}$ & $\begin{array}{l}a_{m}=5.911(1), b_{m}=26.103(5) \\
\quad c_{m}=11.874(2), \beta_{m}=106.11(3)\end{array}$ & $\begin{array}{l}a_{M}=5.951(1), b_{M}=25.223(5) \\
\quad c_{M}=23.593(4), \beta_{M}=103.60(2)\end{array}$ \\
\hline Crystal system & Orthorhombic & Monoclinic & Monoclinic \\
\hline Space group & $B m m b$ & $P 2_{1} / c$ & $P 2_{1} / c$ \\
\hline Multiplicity & 4 & 4 & 8 \\
\hline$R_{1}\left[F_{o}>4 \sigma\left(F_{o}\right)\right]$ & 0.0405 & 0.0363 & 0.0459 \\
\hline \multirow[t]{3}{*}{ Comments } & Two chains superimposed & Two orientational domains & Two orientational domains \\
\hline & Disordered acid group & One chain conformation & One chain conformation \\
\hline & & $\begin{array}{l}\text { Disordered acid group volume } \\
\text { ratios: } \alpha=0.88 / \beta=0.12\end{array}$ & $\begin{array}{l}\text { Ordered acid group volume ratios: } \\
\qquad \alpha=0.67 / \beta=0.33\end{array}$ \\
\hline
\end{tabular}

process was so fast that we could not observe the (401) domains. The presence of both (401) and (401) domains in Fig. 3(a) proves the ferroelastic nature of the transition process. Figs. 3(b), 3(c) and 3(d) allow a better visualization of the propagation of the phase front. The II $\rightarrow$ III transition begins with the formation of lancet-shaped (401) domains at the [101] edge of the crystal (Fig. 3b). These gradually pierce the whole crystal towards the (101) edge. A few moments later these (401) needles are transformed into the already mentioned (100) domains. The phase front (black line) associated with this process is rather fuzzy and may be approximated by (001); the front's advancement is indicated by the black arrow (Figs. $3 b$ and $3 c$ ). Fig. $3(d)$ illustrates the III $\rightarrow$ II transition. This figure shows the total reversibility of the process: the (100) domains give way to the $(40 \overline{1})$ domains and finally phase II is reestablished. The III $\rightarrow$ II transition is again initiated by a (001) phase front that transforms into a hybrid type of phase front (white line). The domain sizes and their dynamics vary from crystal to crystal, as expected. This behaviour certainly depends on experimental conditions such as temperature gradient, sample quality and thickness. These observations lead us to postulate that the II $\rightarrow$ III transition is actually a two-step process, which, henceforth, we shall denote II $\rightarrow f \rightarrow$ III. The fact that this fascinating result was not revealed by either diffraction or calorimetry demonstrates the value of orthoscopy. The angle between the extinction directions in the two domains in phase III was estimated to lie between 2 and $4^{\circ}$. In view of the experimental uncertainties (domains measure $\sim 1 \mu \mathrm{m}$ ) this result represents a satisfactory agreement with the spontaneous deformation, which is computed as twice the angle between $[201]_{m}$ and [001] of $2^{\circ}$.

On cooling further, at $T_{\mathrm{III} \rightarrow \mathrm{IV}}$ another phase front crosses the crystal from one [101] edge to the corresponding edge on the other side of the platelet (Fig. 4). This front can also emerge at the [101] edge but not always on the same edge in subsequent cycles. The orientational (100) domains are no longer seen, but some rather large and deep cracks and furrows appear on the (010) face, their direction being [001]. In some crystals the transition is particularly violent and whole chips are torn out of the core. No further major changes occur down to $\sim 183 \mathrm{~K}$. When reheating, no visual phenomena are
Table 3

Some characteristics of the two simplest MDOs.

\begin{tabular}{|c|c|c|c|c|c|}
\hline & Nickname & Maxima & Cell & & Space group \\
\hline $\mathrm{MDO}_{1}$ & Screwed & $\xi=\frac{1}{2}, 1, \frac{3}{2}$ & $\begin{array}{l}\mathbf{a}_{1}=2 \mathbf{a}_{o} \\
\mathbf{b}_{1}=\mathbf{b} \\
\mathbf{c}_{1}=\mathbf{c}\end{array}$ & & $P c c 2$ \\
\hline $\mathrm{MDO}_{2}$ & Slated & $\xi-\frac{\ell}{4} \in Z$ & $\begin{array}{l}\mathbf{a}_{2}=-\mathbf{a}_{o}+\mathbf{c} / 4 \\
\mathbf{b}_{2}=\mathbf{b} \\
\mathbf{c}_{2}=-\left(2 \mathbf{a}_{o}+\mathbf{c} / 2\right)\end{array}$ & $\beta \simeq 106^{\circ}$ & $P 2_{1} / c$ \\
\hline
\end{tabular}

detected up to $\sim 282 \mathrm{~K}$, at which temperature some, but not all, of the fissures seem to heal. Further cooling and heating cycles confirm this sequence of events, except that the III $\rightarrow$ IV phase front starts sometimes at the [101] edge and

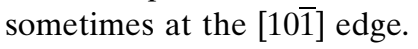

\subsection{X-ray diffraction}

2.5.1. Bragg reflection data and reciprocal space survey. A shiny, translucent, colourless, rhomb-shaped crystal was glued onto a thin glass fibre and kept at 286 (1) $\mathrm{K}$ in a stream of nitrogen gas during data collection, which took place on a Stoe IPDS system using Mo $K \alpha$ radiation. (Note that, once out of the stream, the crystal surfaces became infested with whitishopaque stains in a matter of hours; we presumed that these stains were due to solvent loss.) The image-plate-to-crystal distance was set to $60 \mathrm{~mm}$ and a $\varphi$ interval of $1^{\circ}$ was chosen. Two hundred images were then exposed for $5 \mathrm{~min}$ each. The integration was carried out using profiles of between 15 and 11 pixels and an effective mosaic spread of 0.013 . (The unusual profile function stems from the diffuse scattering; see below). The intensities were corrected for Lorentz and polarization effects, but no absorption correction was deemed necessary. An inspection of reciprocal space revealed that most of the maxima found in the images could be indexed in the cell given in Table 2; the remaining maxima lay on lines $\left(\xi, k, l+\frac{1}{2}\right)$, $\xi \in R$ and $k, l \in Z$. These rods and other more complex regions of diffuse scattering have also been observed on precession photographs recorded using $\mathrm{Cu} K \alpha$ radiation. In lieu of such a photograph we display a reconstruction of the 


\section{research papers}

$(\xi, 4, l)$ layer (Fig. $5 a)$ from the oscillation diffractograms of the image-plate data collection. This figure shows clearly the rods of diffuse scattering along $\mathbf{a}^{*}$. A closer inspection of this diffraction pattern immediately reveals that it may be inter- preted to a good approximation as stemming from an OD structure as defined by Dornberger-Schiff (1966). The whole diffraction pattern is found to be indexable based on a layer group $P(c) c 2$ and a cell $a_{o}=4.708(1), b=26.124$ (5) and $c=14.406$ (2) $\AA$. Further inspection of the diffuse lines reveals two classes of weak maxima within the lines, namely $\quad\left(h+\frac{1}{2}, k, l+\frac{1}{2}\right) \quad$ and $\left(h, k, l+\frac{1}{2}\right), h, k, l \in Z$. These two classes of reflections correspond to the simplest maximal degree of order (MDO) structures in this OD structure (Table 3). The latter $\left(h, k, l+\frac{1}{2}\right)$ corresponds to $\mathrm{MDO}_{1}$, which is realized in phase II of UA but is completely crystallized in urotropin pimalate (Gardon, Pinheiro \& Chapuis, 2003), whereas $\mathrm{MDO}_{2}$ corresponds exactly to phase III (Hostettler et al., 1999) of UA. Phase II of UA is thus found to be a mixture of the two simplest MDOs at $286 \mathrm{~K}$. The $(\xi, 4, l)$ layer of phase III is displayed in Fig. 5(b). A detailed analysis reveals that this layer can be understood as arising from a stacking fault created by the $C_{2 x}$ partial operation that leads to twin domains described in the monoclinic cell $\mathbf{a}_{m}=-\mathbf{a}_{o}+\mathbf{c} / 4, \quad \mathbf{b}_{m}=\mathbf{b}, \quad \mathbf{c}_{m}=$ $-2\left(\mathbf{a}_{o}+c / 2\right)$ and in the cell equiva-
Figure 3

Two typical (010) platelets of UA, $(a)$ and $(b, c, d)$, as seen under crossed Nicols. White double-arrows indicate (401) and (401) domains, black double-arrows (100) domains, while white and black lines emphasize the phase fronts.
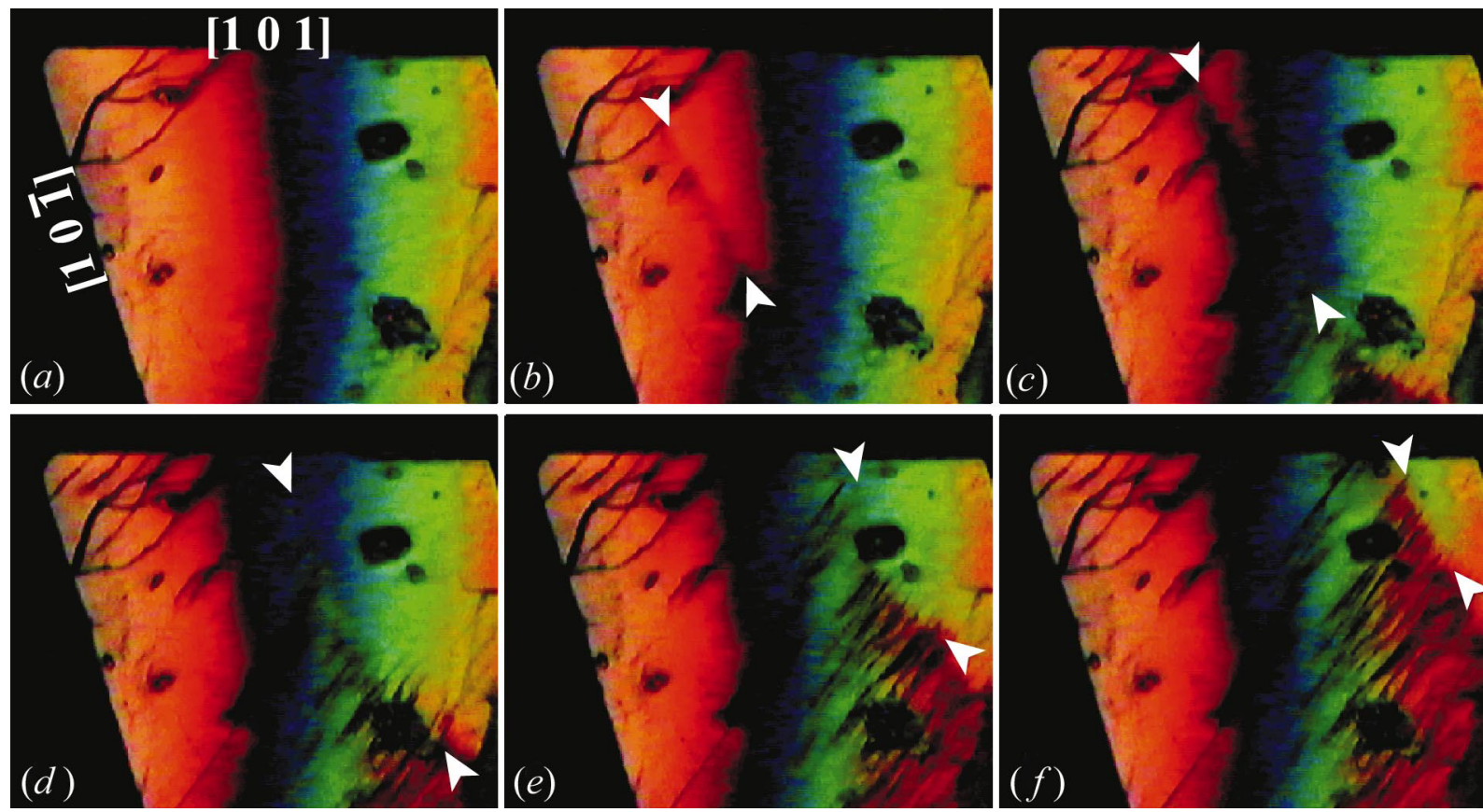

Figure 4

(010) platelet of UA as seen under crossed Nicols. (a) Displays the platelet shortly before the II $\rightarrow$ IV transition, $(b)-(f)$ show the progress of the (101) phase front (best recognized between the white arrows) during the transition and the ensuing damage to the crystal. 

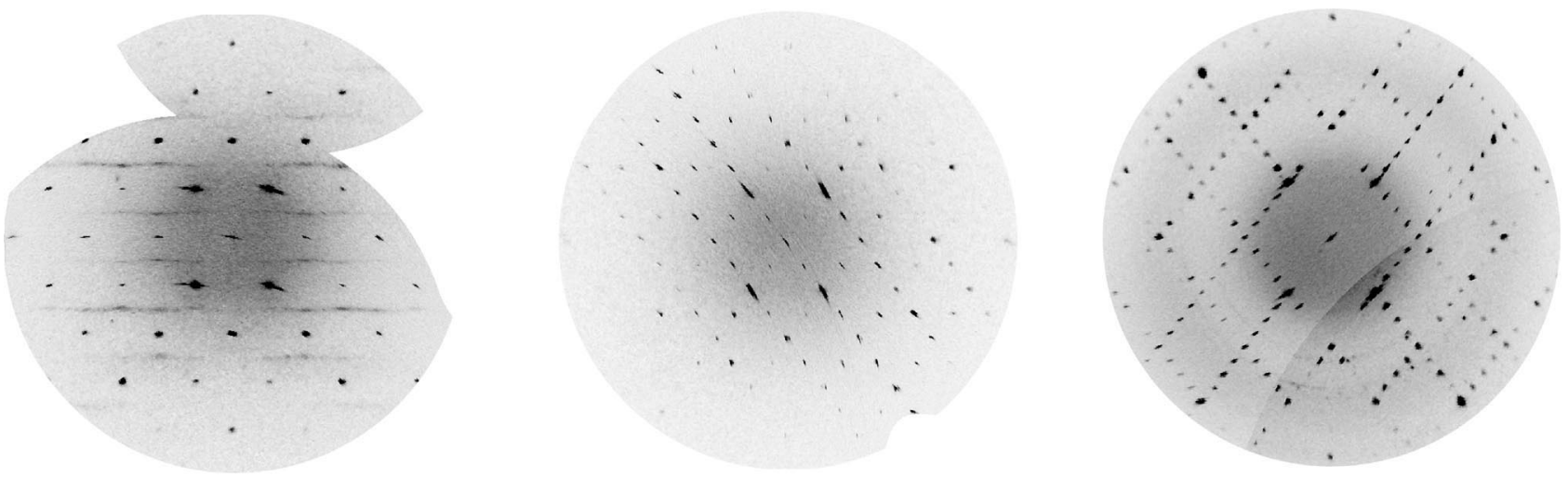

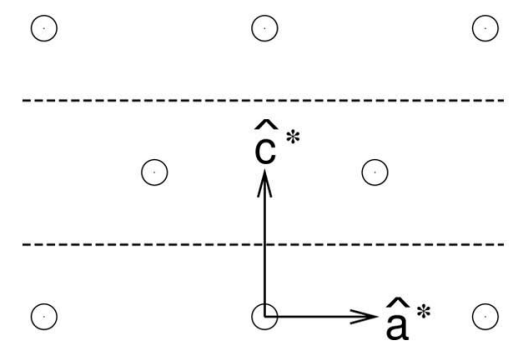

II

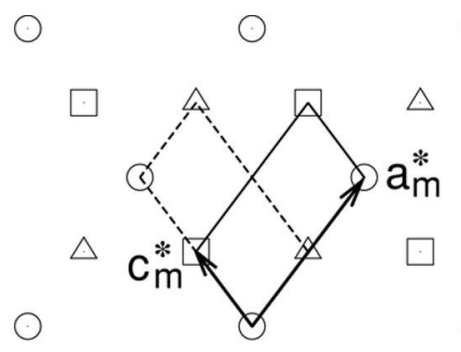

III

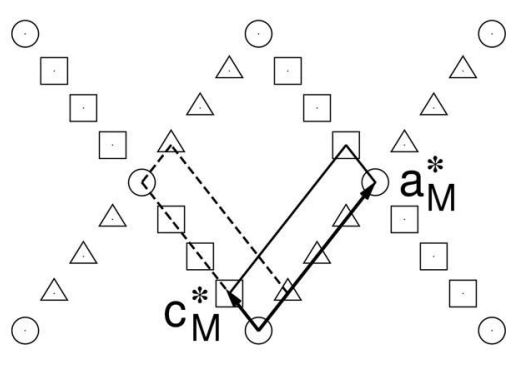

IV

Figure 5

$(\xi 4 \ell)$ layers of phase II, phase III and phase IV reconstructed from 200 images with the program SPACE (Stoe \& CIE GmbH, 1997).

lent to it by the action of $\sigma_{x}$.

Fig. 5(c) shows the $(\xi 4 l)$ diffraction pattern of phase IV (at $200 \mathrm{~K}$ ). This phase, which is the ground state of the system, is completely ordered (Birkedal et al., 2003) and is clearly most interesting. Not only is this phase none of the simplest MDO structures, but the transition towards it is associated with a point in the interior of the Brillouin zone of phase II.

The structure of phase II was solved by direct methods (Sheldrick, 1997b) and refined using SHELXL (Sheldrick, 1997a). The $\mathrm{U}$ molecules lie on the $C_{2 v}$ site, which is a subgroup of the full $T_{d}$ symmetry of U: no problems there. The AA chains, however, turn out to lie perpendicular to the (100) plane. Since their long axes make an angle of $54^{\circ}$ with the $b$ axis, they do not occupy the only site compatible with their natural $C_{2 v}$ symmetry: there are two disordered half-chains related by $\sigma_{x}$. This result is not at all surprising since we are considering the superposition structure. This structure contains unrealistic atomic positions, which must then be 'distributed' in order to obtain the true OD structure. Each disordered chain contains two disordered carboxyl groups, because there are two hydrogen-bond donor atoms available. The methylene $\mathrm{H}$ atoms were inserted at appropriate positions relative to their associated $\mathrm{C}$ atoms, upon which the $\mathrm{H}$ atoms were made to ride. However, the acid proton was found in a difference map and naively refined with $x, y, z$ and $\mathrm{U}$. It reached a position about halfway between $\mathrm{O}_{1 b}$ and $\mathrm{N}_{2}$. (a)
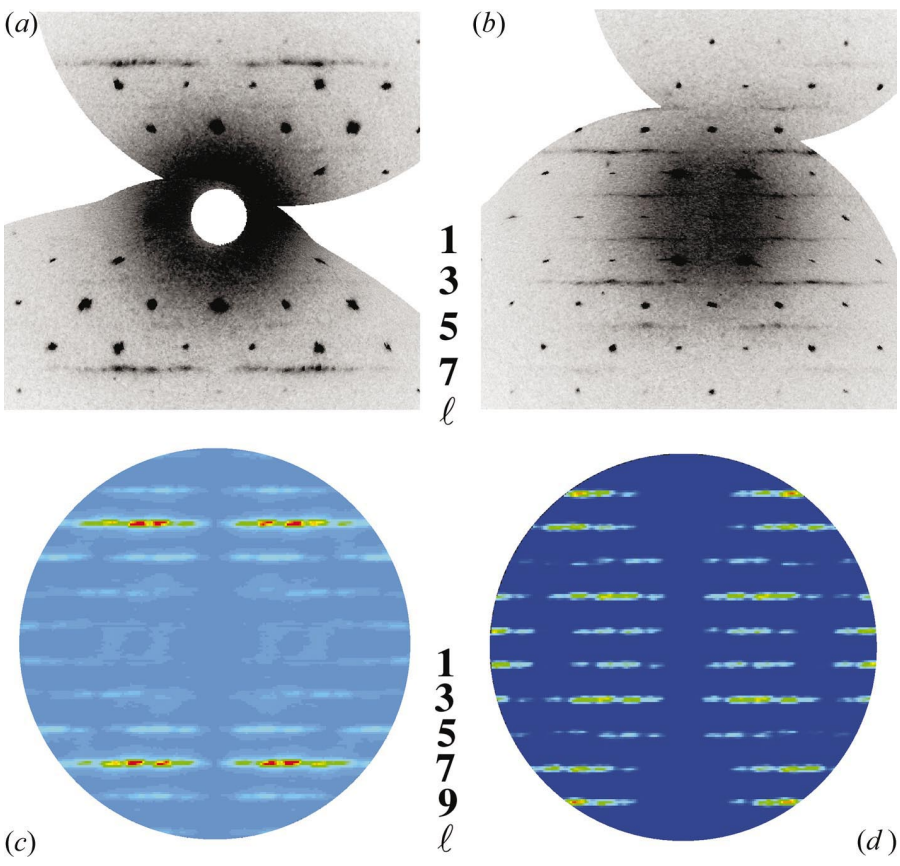

\section{Figure 6}

(a) $(\xi 1 \ell)$ and $(b)(\xi 4 \ell)$ layers reconstructed from 200 images with the program SPACE (Stoe \& CIE GmbH, 1997). (c) ( $\xi 1 \ell)$ and $(d)(\xi 4 \ell)$ layers simulated using correlations $C_{1}^{a} \simeq 0.6$ and $C_{2}^{a} \simeq 0.2$. The temperature factor fall-off is not taken into account and Bragg spots have been eliminated in the calculations for the sake of clarity. 
Table 4

Experimental details for phase II of UA.

\begin{tabular}{|c|c|}
\hline Chemical formula & $\mathrm{C}_{6} \mathrm{H}_{12} \mathrm{~N}_{4} \cdot \mathrm{C}_{9} \mathrm{H}_{16} \mathrm{O}_{4}$ \\
\hline Chemical formula weight & 328.41 \\
\hline Cell setting, space group & Orthorhombic, Bmmb \\
\hline$a, b, c(\AA)$ & 9.4157 (19), 26.124 (5), 7.2034 (14) \\
\hline$V\left(\AA^{3}\right)$ & $1771.9(6)$ \\
\hline$Z$ & 4 \\
\hline$D_{x}\left(\mathrm{Mg} \mathrm{m}^{-3}\right)$ & 1.231 \\
\hline Radiation type & Mo $K \alpha$ \\
\hline $\begin{array}{l}\text { No. of reflections for cell para- } \\
\text { meters }\end{array}$ & 721 \\
\hline$\theta$ range $\left(^{\circ}\right)$ & $14.5-24.1$ \\
\hline$\mu\left(\mathrm{mm}^{-1}\right)$ & 0.09 \\
\hline Temperature (K) & $286(1)$ \\
\hline Crystal form, colour & Platelet, colourless \\
\hline Crystal habitus & $\{101\},\{10 \overline{1}\}$ and $\{010\}$ pinacoids \\
\hline Crystal size $(\mathrm{mm})$ & $0.72 \times 0.56 \times 0.04$ \\
\hline Diffractometer & Stoe IPDS \\
\hline Data collection method & $\varphi$ scans \\
\hline Absorption correction & None \\
\hline$T_{\min }$ & - \\
\hline$T_{\max }$ & - \\
\hline $\begin{array}{l}\text { No. of measured, independent and } \\
\text { observed parameters }\end{array}$ & $6062,818,539$ \\
\hline Criterion for observed reflections & $I>2 \sigma(I)$ \\
\hline$R_{\text {int }}$ & 0.053 \\
\hline$\theta_{\max }$ & 25 \\
\hline$F(000)$ & 712 \\
\hline Range of $h, k, l$ & $\begin{array}{l}-10>h>10 \\
-31>k>31 \\
-8>l>8\end{array}$ \\
\hline Refinement on & $F^{2}$ \\
\hline$R\left[F^{2}>2 \sigma\left(F^{2}\right)\right], w R\left(F^{2}\right), S$ & $0.041,0.126,0.91$ \\
\hline $\begin{array}{l}\text { No. of reflections and parameters } \\
\text { used in refinement }\end{array}$ & 818,110 \\
\hline $\mathrm{H}$-atom treatment & Mixed \\
\hline Weighting scheme & $\begin{array}{l}w=1 /\left[\sigma^{2}\left(F_{o}^{2}\right)+(0.0754 P)^{2}+\right. \\
\quad 0.4007 P] \text { where } P=\left(F_{o}^{2}+2 F_{c}^{2}\right) / 3\end{array}$ \\
\hline$(\Delta / \sigma)_{\max }$ & $<0.001$ \\
\hline$\Delta \rho_{\max }, \Delta \rho_{\min }\left(\mathrm{e} \AA^{-3}\right)$ & $0.15,-0.13$ \\
\hline
\end{tabular}

Computer programs: Stoe IPDS (Stoe \& CIE GmbH, 1997); SHELXS97 (Sheldrick, 1997b); SHELXL97 (Sheldrick, 1997a).

The final refinement results are given in Table 4, together with additional information on the diffraction experiment. Releasing the $\mathrm{C}-\mathrm{H}$ distance in the $\mathrm{CH}_{2}$ groups did not improve the residuals. Fractional coordinates of the final model are given in the supplementary material. ${ }^{\mathbf{1}}$

2.5.2. PSD diffuse-scattering data. Although the reciprocal space images shown in Fig. 6 were obtained with the imageplate system that was used for measuring the Bragg reflection data, we also carried out diffraction experiments on the dedicated Weißenberg position-sensitive detector (PSD) diffractometer (Osborn \& Welberry, 1990), which was designed especially for recording diffuse-scattering data. This system is capable of recording complete reciprocal lattice sections with a high resolution and low noise. Two examples of the data recorded in the present study are shown in Fig. 7. The quality of the patterns obtained in the present experiment is much poorer than would normally be expected from this instrument, although the patterns do show more detail than

\footnotetext{
${ }^{1}$ Supplementary data for this paper are available from the IUCr electronic archives (Reference: LC0055). Services for accessing these data are described at the back of the journal.
}

Table 5

Normalized hydrogen-bonding geometry $\left(\AA{ }^{\circ}\right)$.

\begin{tabular}{lllll}
\hline$D-\mathrm{H} \cdots A$ & $d(D-\mathrm{H})$ & $d(\mathrm{H} \cdots A)$ & $d(D \cdots A)$ & $<(D-\mathrm{H} \cdots A)$ \\
\hline $\mathrm{O} 1-\mathrm{H} 1 \cdots \mathrm{N} 1$ & $1.34(5)$ & $1.56(7)$ & $2.732(4)$ & $140.2(19)$ \\
$\mathrm{C} 7-\mathrm{H} 7 a \cdots \mathrm{O} 2 b^{\mathrm{i}}$ & 1.092 & 2.53 & $3.240(12)$ & 121.6 \\
$\mathrm{C} 7-\mathrm{H} 7 b \cdots \mathrm{O} 2 a^{\mathrm{i}}$ & 1.092 & 2.69 & $3.186(14)$ & 107.1 \\
$\mathrm{C} 8-\mathrm{H} 8 a \cdots \mathrm{O} 2 b^{\mathrm{ii}}$ & 1.092 & 2.30 & $3.349(11)$ & 160.3 \\
$\mathrm{C} 9-\mathrm{H} 9 a \cdots \mathrm{N} 2^{\mathrm{iii}}$ & 1.092 & 2.65 & $3.654(2)$ & 153.0 \\
$\mathrm{C} 2-\mathrm{H} 2 b \cdots \mathrm{O} 1$ & 1.092 & 2.66 & $3.730(5)$ & 165.1 \\
\hline
\end{tabular}

Symmetry codes: (i) $\frac{1}{2}+x, y, \frac{1}{2}+z$; (ii) $x, \frac{1}{2}-y, 1+z$; (iii) $-\frac{1}{2}+x, y,-\frac{1}{2}+z$; (iv) $\frac{3}{2}-x, y$, $-\frac{1}{2}+z$.

the images of Fig. 6. The main reasons for this poor quality are the presence of the powder rings, streaking emanating from the strong Bragg peaks and the generally high levels of parasitic scattering that may be seen. Despite repeated attempts to obtain clean high-quality samples it was invariably found that during the course of the $\sim 3 \mathrm{~d}$ required to record each section the quality of the crystal deteriorated and the powder rings and other parasitic scattering effects could not be avoided. Further experiments are in progress to try to improve on the quality of these data.
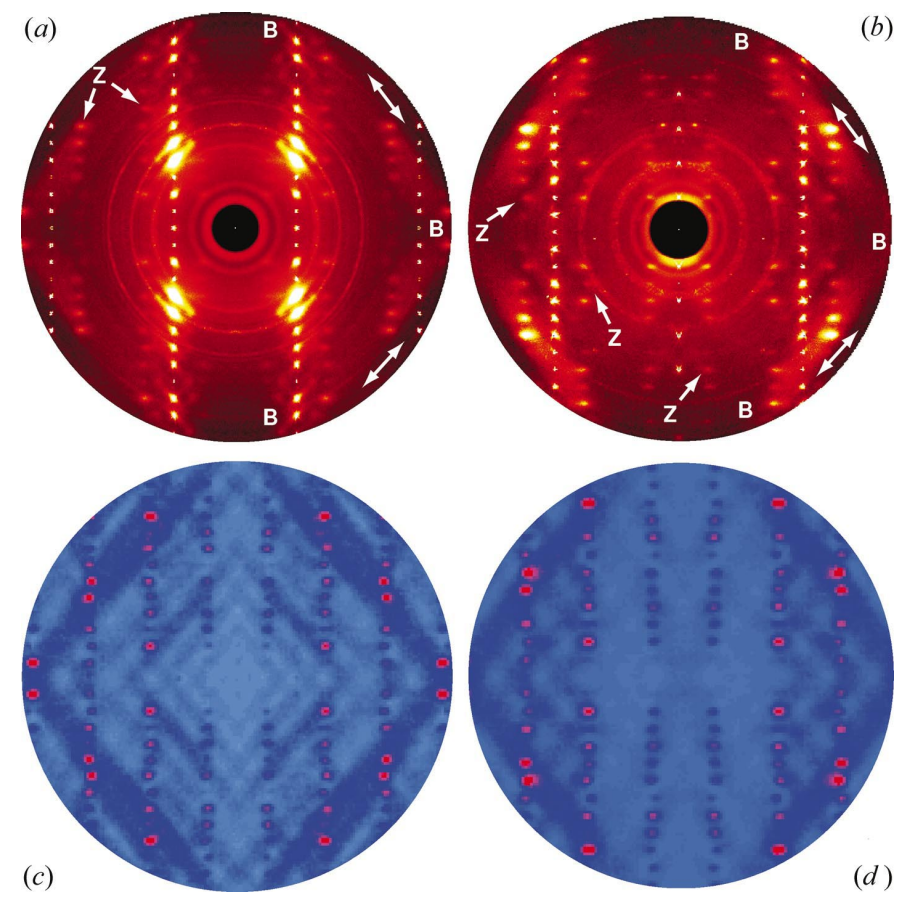

(b)

\section{Figure 7}

(a) $(1 k \ell)$ and $(b)(2 k \ell)$ layers recorded on the Wei $\beta$ enberg PSD

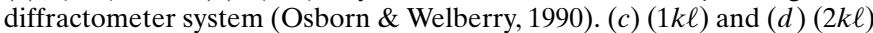
layers simulated with the help of the program DIFFUSE using correlations $C_{1}^{a} \simeq 0.6, C_{2}^{a} \simeq 0.2, C_{1}^{b} \simeq-0.7$ and $C_{1}^{c} \simeq-0.7$. Bragg spots have been omitted in the calculations for the sake of clarity and temperature factor fall-off has been neglected. 


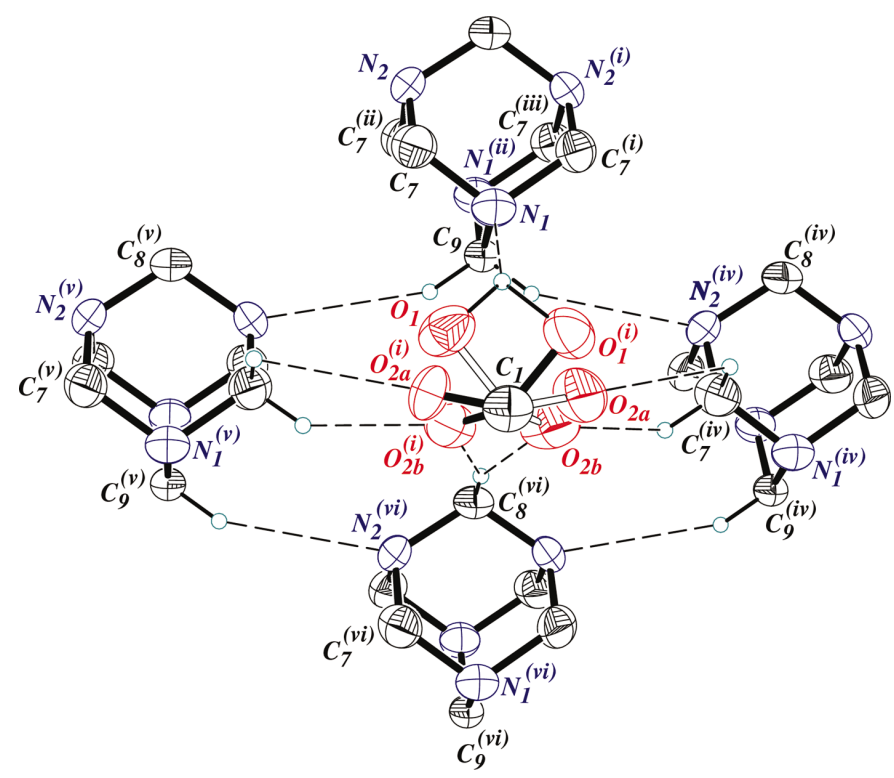

Figure 8

The hydrogen bonds involved in the packing of UA in Phase II. ${ }^{(i)}$ stands for $\sigma_{x} \mid 100,{ }^{(i i)}$ for $\sigma_{y} \mid 0 \frac{1}{2} 0,{ }^{(i i i)}$ for $C_{2} z \mid 1 \frac{1}{2} 0,{ }^{(i v)}$ for $E \mid \frac{1}{2} 0 \frac{1}{2},{ }^{(v)}$ for $E \mid \frac{1}{2} 01 \frac{1}{2}$ and ${ }^{(v i)}$ for $E \mid 00 \hat{1}$. Atoms are drawn at the $30 \%$ probability level and only pertinent $\mathrm{H}$ atoms are plotted for the sake of clarity.

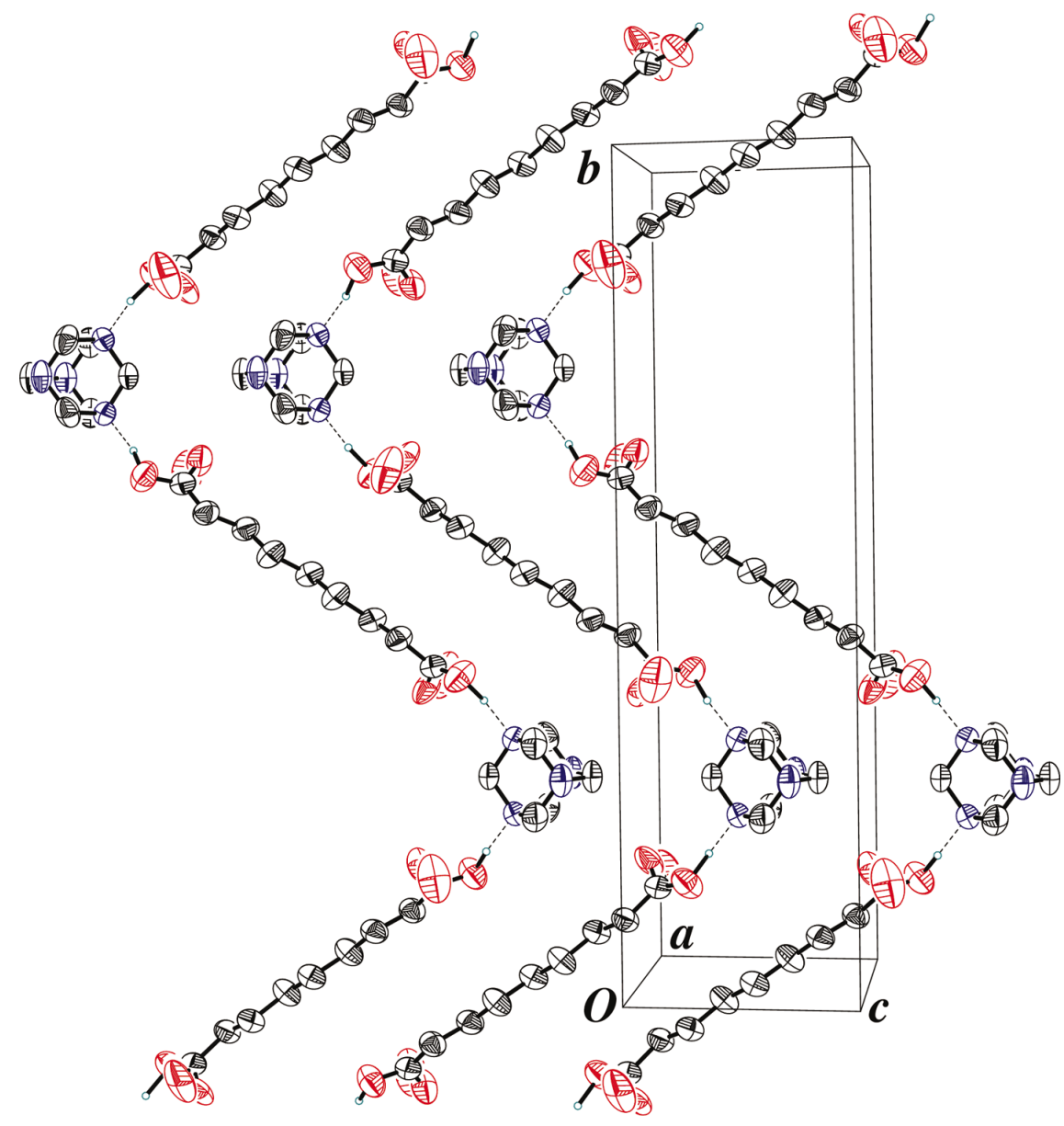

Figure 9

(100) layer of phase II of UA. Only one of the two disordered chains is shown, so the view represents the layer group $P(c) c 2$ as well. The next layer (before or behind) would be shifted by $\widehat{\mathbf{c}} / 2$.

\section{Discussion}

\subsection{The superposition structure}

UA is a co-crystal of $U$ balls and AA sticks. In view of the alternating (010) sheets of balls and sticks, it might be classified as a layer structure for the sake of chemical homogeneity. However, the weakness of the bonding between successive (100) sheets suggests that it is the latter that will turn out to be the important layers for most if not all of the extraordinary phenomena observed in UA. Five different hydrogen bonds play a role in UA (Table 5, Fig. 8). Their normalized distances and angles all lie within the accepted ranges (Jeffrey \& Sänger, 1991; Steiner \& Desiraju, 1998). One bond even qualifies as strong, since the refined $\mathrm{H}$ atom lies about halfway between $\mathrm{N}_{2}$ and $\mathrm{O}_{1 b}$. The pertinent $\mathrm{N}_{2}-\mathrm{H}-\mathrm{O}_{1 b}$ angle is a little on the small side, but it appears nevertheless to play a crucial role in the phase transitions and other phenomena that occur in UA. The (100) layers are connected by one weak $\mathrm{C}-\mathrm{H} \cdots \mathrm{O}=\mathrm{C}$ bond, one esoteric $\mathrm{C}-\mathrm{H} \cdots \mathrm{N}$ hydrogen bond and van der Waals forces, whereas the (010) layers are held together by one strong $\mathrm{N} \cdots \mathrm{H} \cdots \mathrm{O}$ and three weak $\mathrm{C}-\mathrm{H} \cdots \mathrm{O}=\mathrm{C}$ hydrogen bonds. The sometimes discredited $\mathrm{C}-\mathrm{H} \cdots \mathrm{O}=\mathrm{C}$ hydrogen bond is indisputably effective in determining the packing of UA. Its approximate energy of $2 \mathrm{kcal} \mathrm{mol}^{-1}$ (Jeffrey \& Sänger, 1991) is certainly comparable with the sum of van der Waals energies of the various methylene groups [the typical energy of which is roughly $0.3 \mathrm{kcal} \mathrm{mol}^{-1}$ for methylene groups $4 \AA$ apart (Salem, 1962)].

The AA chains all lie with their long axes perpendicular to [100] such that the axes make an angle of $54^{\circ}$ with the $b$ axis. Within any one (010) layer the molecular planes defined by their zig-zag backbone are all parallel to each other and perpendicular to the (100) plane. The $\left\{C_{2 y} \mid 0 \frac{1}{2} 0\right\}$ axis causes successive layers of chains to turn by $180^{\circ}$ such that the chains are arranged in a herringbone-like pattern when viewed along the $a$ axis (Fig. 9). The packing of the AA chains may best be approximated by Abrahamson's $p$-cell $\mathrm{O}_{\|}$ (Abrahamsson et al., 1978), although this is actually a gross idealization. In UA we find for this $p$-cell $a=9.42, b=4.12$, $c=2.50 \AA$, but the angles clearly differ from $90^{\circ}$, especially $\beta$. The typical chainchain distances in UA start at $\sim 4.10 \AA$ and the area per methylene group is $19.1 \AA^{2}$. [Below we compare some values with those of a typical long-chain structure, $n$-tetradecylammoniumchloride $(\mathrm{C} 14 \mathrm{Cl})$, at room temperature (Schenk, 1984).] In this almost ideal structure the area per methylene group is $18.3 \AA^{2}$ and the chain-chain distances are about $0.1 \AA$ 


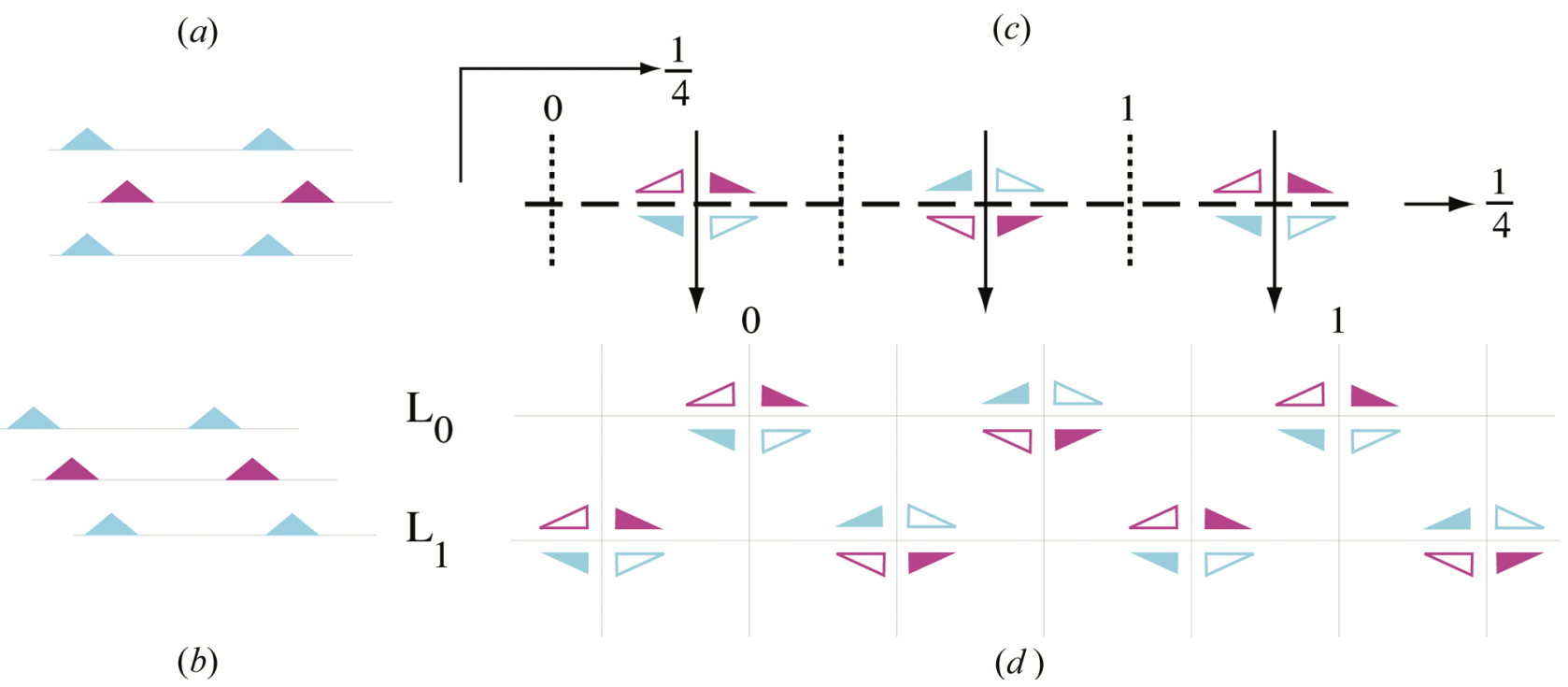

Figure 10

(a) The 'screwed' triple (think of the profile of a screw). (b) The 'slated' triple (reminiscent of the steps in a slab of the mineral slate). (c) The layer group $P(c) c b .(d)$ The gruppoid family No. 27 of Dornberger-Schiff (1966).

shorter than in UA. These facts indicate that the chains are not as densely packed in UA as in a comparable alkane or alkanedioic acid structure. It appears that $\mathrm{U}$ is the determining factor for the crystal packing.

The geometry of the AA chains is rather far from normal. In UA the $\mathrm{C}-\mathrm{C}$ distances, $\mathrm{C}-\mathrm{C}-\mathrm{C}$ angles and $\mathrm{C}-\mathrm{C}-\mathrm{C}-\mathrm{C}$ torsion angles are $1.516(8) \AA, 114(2)$ and $175(1)^{\circ}$, respectively. For $\mathrm{C} 14 \mathrm{Cl}$ the corresponding values are 1.512 (6) $\AA$, $114.4(6)$ and $179.4(4)^{\circ}$, respectively, and the angles of the $p$ cell are within $0.3^{\circ}$ of the expected $90^{\circ}$. With the information that the chain-chain distances in UA are rather long along the $a$ axis, a picture of not very rigid chains emerges, where these chains are possibly subject to kink diffusion or torsional vibrations. This latter subject will be taken up in a forthcoming communication and has been described by other authors (Thalladi et al., 2000). The loose-chain packing is also suitable for solvent inclusions. Indeed, we have shown by means of GC that the UA crystals contain traces of acetonitrile (about one molecule per one hundred cells of UA).

Of the two refined O-atom positions one corresponds to a carboxyl group that lies very roughly in the zig-zag plane of AA $\left(\mathrm{O}_{1 b}-\mathrm{C}_{1}-\mathrm{O}_{1 a 2}\right.$ dihedral angle is $\left.\sim 11^{\circ}\right)$ and the other corresponds to a $\mathrm{COOH}$ group with a dihedral angle of $\sim 40^{\circ}$ $\left(\mathrm{O}_{1 b}-\mathrm{C}_{1}-\mathrm{O}_{1 a 1}\right)$. These values are surprisingly close to those found in the $\alpha$ form of AA. In this phase, which is stable above $75^{\circ}$, one finds angles of 6 and $37^{\circ}$. The geometry of the disordered carboxyl group is not altogether unsatisfactory: the double bond measures 1.10 (1) $\AA$ for $\mathrm{O}_{1 a 1}$ and 1.13 (1) $\AA$ for $\mathrm{O}_{1 a 2}$, whereas the single bond is $1.372(4) \AA$. The corresponding values in the $\beta$ form of AA (Housty \& Hospital, 1967; Bond et al., 2001) are 1.23 and $1.30 \AA$. The $\mathrm{O}-\mathrm{C}-\mathrm{O}_{1 b}$ angles are $123.0(7)^{\circ}$ for $\mathrm{O}_{1 a 1}$ and $115.8(8)^{\circ}$ for $\mathrm{O}_{1 a 2}$; these values are not too different from the angle in $\mathrm{AA}(\beta)$, namely $123.5^{\circ}$. Although our refinement is not completely satisfactory it still reproduces, at least qualitatively, the geometrical characteristics of the pure AA chains. A comprehensive search (Birkedal et al., 2003) of the CSD database has shown that $\mathrm{C}_{\beta}-\mathrm{C}_{\alpha}-\mathrm{O}_{1}-\mathrm{O}_{2}$ is essentially planar and that the sum of the angles is $360(0.5)^{\circ}$. In phase II of UA these values are $0.031 \AA$ and $358.7^{\circ}$ for the $\mathrm{C}_{2}-\mathrm{C}_{1}-\mathrm{O}_{1}-\mathrm{O}_{2 a}$ group and $0.083 \AA$ and

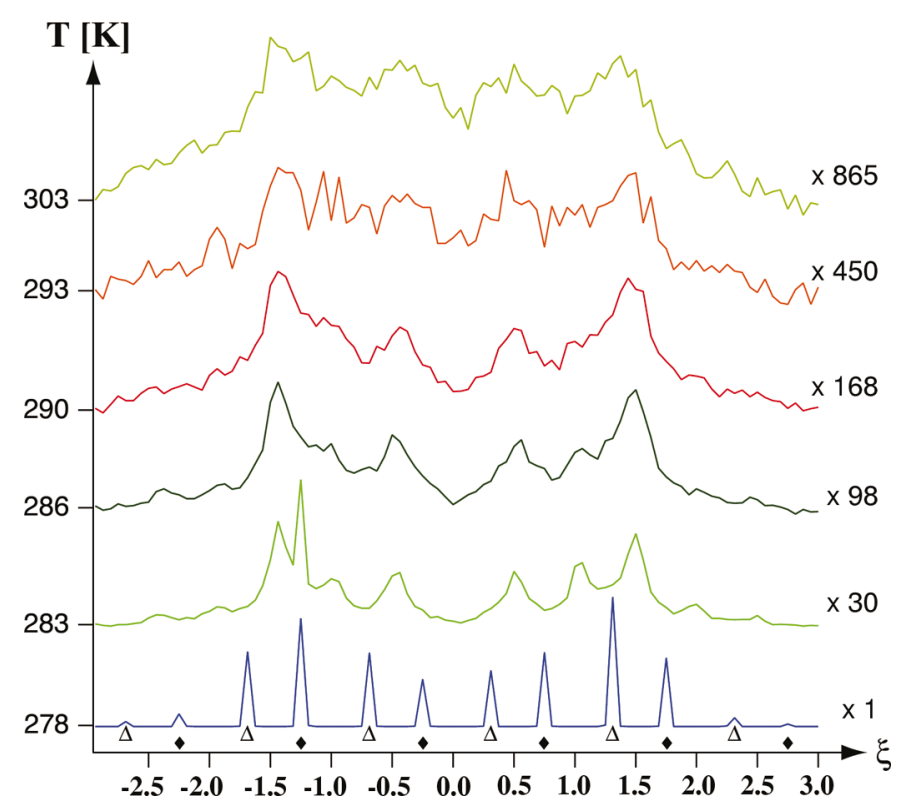

Figure 11

Scan along the diffuse $\operatorname{rod}(\xi, 1,7)$. The $\xi$ index is with respect to the layer separation $\mathbf{a}_{o}$. The topmost scan is an average of five scans on and \pm 0.02 off the line. The intensities are normalized with respect to the scan at $T=278 \mathrm{~K}$ (normalization factors are given at the right end of each scan). The second scan from the bottom sits right on the transition as can be learned from the breakthrough of the line of phase III. Besides the obvious II $\rightarrow$ III transition there is another subtle, but irrefutable, transition within phase II that appears between 293 and $290 \mathrm{~K}$. 
Table 6

Systematic absences in the diffraction pattern.

\begin{tabular}{llll}
\hline & In & There are & If \\
\hline i & $(\hat{h}, k, \hat{l})$ & Sharp reflections & $\hat{h}+\hat{l}$ even \\
ii & $(\hat{h}, k, 0)$ & Sharp reflections & $k$ even \\
iii & $(\xi, k, \ell)$ & Sharp reflections & $\ell=2 \hat{l}$ \\
iv & $(\xi, k, \ell)$ & Diffuse rods & $\ell$ odd \\
v & $(\xi, 0, \ell)$ & No diffuse rods & \\
vi & $(\xi, k, \ell)$ & Vanishing intensities of diffuse rods & $\xi \rightarrow 0$ \\
\hline
\end{tabular}

$351.2^{\circ}$ for the $\mathrm{C}_{2}-\mathrm{C}_{1}-\mathrm{O}_{1}-\mathrm{O}_{2 b}$ group. We have tried to split the $\alpha$ carbon, but the ideal geometry cannot be obtained without restraints. However, in view of the OD character of UA and the quite large anisotropic displacement parameters, these values seem to warrant reasonable chains even at the chain ends. It might, however, be interesting to check with spectroscopic methods whether this non-ideal behaviour is the expression of the fact that the two chains ends might have different degrees of deprotonation (Housty \& Hospital, 1967).

We have said that the packing of the co-crystal seems to be governed by the packing of the U balls. Indeed, the (101) planes of U alone (Kampermann et al., 1995) closely resemble the (010) planes of UA. The translations in the (101) plane are 7.02 and $9.93 \AA$ at $298 \mathrm{~K}$ and the distances between the $\mathrm{U}$ molecules are $6.08 \AA$. The same translations in UA are 7.2034 and $9.416 \AA$ at $286 \mathrm{~K}$ and the distance between U molecules is $5.9 \AA$. U is not a plastic crystal and therefore the $\mathrm{C}-\mathrm{H} \cdots \mathrm{N}$ subunits $\left(1.098 \AA, 2.785 \AA, 151.4^{\circ}\right)$ may be assessed as hydrogen bonds. In UA we have the same situation $(1.092 \AA$, $2.65 \AA$, $153^{\circ}$; Table 5). On formation of the co-crystal the $U$ molecules seem, compared with pure $U$, to have approached each other a little more closely and to have formed slightly stronger hydrogen bonds.

\subsection{The OD structure}

We have already mentioned (see \$2.2) the rods of diffuse scattering that are the most salient features of the diffraction pattern of phase II. Following Guinier's elegant derivation (Guinier, 1964), we conclude that we are dealing with a planar disorder in this phase, i.e. a more or less random sequence of $(\mathbf{b}, \mathbf{c})$ layers along $\mathbf{a}_{o}$. Making use of Käte Dornberger-Schiff's formalism (Dornberger-Schiff, 1966), we shall now give a more complete description of phase II that will account for more of the diffraction pattern than the mere superposition structure.

A more detailed analysis of the said diffraction pattern results in the systematic absences of Table 6 (these are given with respect to a cell $\mathbf{a}_{o}, \mathbf{b}, \mathbf{c}$, where $\mathbf{a}_{o}$ is the layer separation). Condition (iii) tells us that the c base vector of the superposition structure is $\widehat{\mathbf{c}}=\mathbf{c} / 2$ and (i) that the structure is $B$ centred. Absence (ii) reveals that there is a $b$-glide plane perpendicular to c. The global law (i) furthermore includes an $n$-glide plane perpendicular to $\mathbf{b}$ and a $c$-glide plane perpendicular to c. These results amount to the space group $B m m b$ for the superposition structure and the base vectors $\widehat{\mathbf{a}}=2 \mathbf{a}_{o}$, $\widehat{\mathbf{b}}=\mathbf{b}$ and $\widehat{\mathbf{c}}=\mathbf{c} / 2$. According to (v) there are no diffuse rods in $(\xi, 0, \ell)$; the absence $\ell=2$ corresponds to a $c$-glide plane perpendicular to $\mathbf{b}$ in the OD structure. The condition $k=2 n$ in $(\xi, k, 0)$ indicates a $b$-glide plane perpendicular to c. The absence $\ell=2 n$ in $(0, k, \ell)$ results from a $c$-glide plane perpendicular to a. Combining all these $\lambda$ POs (partial operations) leads to the layer group $P(c) c b$, as shown in Fig. 10(c).

The $\widehat{\mathbf{c}}$ base vector of $\mathbf{c} / 2$ reveals that the layer $L_{1}$ is shifted by $\mathbf{c} / 4$ with respect to the layer $L_{o}$. The combination of this displacement, namely $\mathbf{t}=\mathbf{a}_{o}+\mathbf{c} / 4$, with the $\lambda$ POs leads to the following OD gruppoid family:

$$
P \quad \begin{array}{ccc}
(c) & c & b \\
\left\{\left(c_{1 / 2}\right)\right. & n_{1 / 2,2} & \left.n_{2,1}\right\}
\end{array}
$$

This OD gruppoid is represented in Fig. 10(c) and corresponds, in a different setting, to the OD gruppoid family No. 27 of Dornberger-Schiff (1966). The figure also demonstrates that there is only one type of layer in this OD structure. (It does not appear necessary in the context of diffuse scattering to be overly concerned with the presence or absence of an inversion centre in the layer.)

If we write the equipoints of this OD structure

$$
\begin{gathered}
{\left[L_{0}\right]=\left[ \pm(x, y, z), \pm\left(x, \bar{y}, \frac{1}{2}-z\right),\right.} \\
\left. \pm\left(\bar{x}, \frac{1}{2}+y, \frac{1}{2}-z\right), \pm\left(\bar{x}, \frac{1}{2}-y, z\right)\right], \\
{\left[L_{p}\right]=\left(p, 0, \gamma_{p}\right)+\left[L_{0}\right], \alpha_{j}= \pm 1, \gamma_{p}=\sum_{j=1}^{p} \alpha_{j}}
\end{gathered}
$$

and define $\gamma_{p}:=p+2 m_{p}$, we obtain

$$
\begin{aligned}
|S(\xi, \ell)|^{2}= & P^{-2}\left\{P+\sum_{p=1}^{P-2} \sum_{p^{\prime}=p+1}^{P-1} \cos 2 \pi\left[\ell\left(m_{p}-m_{p^{\prime}}\right) / 2\right.\right. \\
& \left.\left.+(\xi+\ell / 4)\left(p-p^{\prime}\right)\right]\right\}
\end{aligned}
$$

for the square of the stacking factor. The difference between the various members of the OD gruppoid family lies in the various values of the parameters $m_{p}$ and $m_{p^{\prime}}$. While, obviously, we cannot know these in any direct way, we can at least derive them for some special OD structures, the so-called MDO structures. In this OD gruppoid family there exist two simplest MDO structures. One is based on 'screwed' layer triples (Fig. 10a), the other on 'slated' layer triples (Fig. 10b). By either simple geometrical considerations (which sine wave has maximal overlap with the MDO structure) or substitution for the $m_{p}$ values in the expression for $|S(\xi, \ell)|^{2}$, we can derive conditions on $\xi$ for the MDO structures. Therefore, if we have sizeable regions in the OD structure that have crystallized according to one MDO structure or the other, we shall find pronounced and broad maxima in the diffuse rods at the positions indicated in Table 3. In order to study this point further we undertook to record so-called $Q$-scans for phase II. They were performed on a SYNTEX P3 diffractometer equipped with $\mathrm{Cu} K \alpha$ radiation. The temperature was controlled by means of a stream of nitrogen gas. In Fig. 11 we recognize three types of $Q$-scans: 
(i) The scans at $T=303$ and $293 \mathrm{~K}$ correspond to an OD structure with a rather random layer sequence. Both triples are present in almost equal percentages and no large region of either MDO structure is present.

(ii) The scans at $T=290,286$ and $283 \mathrm{~K}$ present maxima for $\xi=\frac{1}{2}, 1, \frac{3}{2}, 2, \ldots$, those at $\xi= \pm \frac{3}{2}$ being the highest. From the analysis above we know that these maxima stem from the 'screwed' MDO structure.

(iii) The scan at $T=278 \mathrm{~K}$ displays maxima at $\xi-\ell / 4 \in Z$. These maxima are due to the 'slated' MDO structure (phase III). This qualitative assessment is fully corroborated by a structural study (Hostettler et al., 1999). The two ferroelastic domains of this phase are indicated by open triangles and diamonds.

At $303 \mathrm{~K}$ the stacking sequence seems to be fairly arbitrary, although some very broad maxima at $\xi= \pm \frac{1}{2}$ and $\xi= \pm \frac{3}{2}$ may be perceived. There are 'screwed' and 'slated' MDO structures in almost equal proportion, but there is no larger region of either of them. At $293 \mathrm{~K}$, the situation is pretty much the same, but we may already detect some narrowing of the maxima mentioned above. However, between 293 and $290 \mathrm{~K}$, a fundamental change occurs in the OD structure: the peaks have very clearly narrowed to about one third of their FWHM at $293 \mathrm{~K}$ and the maxima at $\xi= \pm \frac{3}{2}$ have doubled in intensity with respect to those at $\xi= \pm \frac{1}{2}$. We shall henceforth refer to the phase above $T_{\alpha}=292 \mathrm{~K}$ as $\alpha$ and to that below $T_{\alpha}$ as II. This tendency continues down to $283 \mathrm{~K}$, the temperature of the II $\rightarrow$ III transition. During this transition all the maxima at $\xi= \pm \frac{1}{2}, \pm 1$ and $\pm \frac{3}{2}$ disappear and new maxima at $-\frac{9}{4},-\frac{5}{4},-\frac{1}{4}, \frac{3}{4}, \frac{7}{4}$ and $\frac{11}{4}$ begin to bulge. These results yield the following picture: At $303 \mathrm{~K}$, both types of triples are present. The regions of the MDO structures are not very large at all. At $292 \mathrm{~K}$, a very diffuse, but irrefutable, phase transition takes place during which the regions of the $\mathrm{MDO}_{1}$ structure suddenly increase in dimension. Obviously, although the maxima that correspond to the screwed MDO are more conspicuous, there are also portions of the slated type in the OD structure. Down to the II $\rightarrow$ III transition, the $\mathrm{MDO}_{1}$ regions become larger until at $T_{\mathrm{II} \rightarrow \mathrm{III}}$ the $\mathrm{MDO}_{1}$ surprisingly switches over to the 'slated' type. It is as if the OD structure had planned to adopt a fully screwed MDO (like it does in UP; Gardon, Pinheiro \& Chapuis, 2003), but suddenly a different thermodynamic path imposed itself towards the slated MDO.

The II $\rightarrow$ III transition is weakly of first order and most puzzling, not only because the system suddenly changes its mind, but also because the transition takes place in two steps. (We will refer to the furtive phase between phases II and III as phase $f$ ). This two-step nature of the transition is reminiscent of the triggered variant of phase transition pioneered by Holakovský (1973), a very interesting example of which has been observed (Bonin et al., 1999). Note that there is no trace at all of this phase transition in the DSC curve. Incidentally, this feature is one of the more salient features of phase II (which according to calorimetry is stable between 283 and $\sim 363 \mathrm{~K}$ ), namely that it undergoes subtle changes again and again. So far we have counted four clearly distinguished subphases within the original phase II. These and the asso- ciated phenomena will be described in a forthcoming paper (Gardon, Schenk et al., 2003). Recently a refined optical experiment conceived by Glazer (Wood \& Glazer, 1980; Geday \& Glazer, 2002) provided some independent evidence of the $\alpha \rightarrow$ II transition. Indeed, $|\sin \delta|$ and $\varphi$ images display $(101) /(10 \overline{1})$ phase fronts at roughly $293 \mathrm{~K}$. Even more intriguingly, the same phase fronts also appear shortly before the II $\rightarrow$ III transition and may therefore be associated with the creation and annihilation of the screwed MDO structure.

\subsection{Beyond the OD structure}

We have mentioned before that the diffraction pattern of UA is more complex than that of a mere OD structure. Indeed, Figs. 7(a) and 7(b) reveal a few special features: rhomb-shaped $(B)$ and cross-shaped [e.g. in ( $3 k \ell)$; not shown] bands of diffuse intensities and deviations from half-integer values of the index $\ell$ of the diffuse rods $(Z)$. A comparison of this figure with Plate 24 of the Atlas of Optical Transforms (Harburn et al., 1975) suggested that these crosses and bands might be related to an occupational disorder of the AA chains. Such a disorder can easily be imagined as growth disorder, since black and white chains occupy strictly the same volume and since the adhesion energies along the $\mathbf{c}$ direction are only very weak van der Waals energies.

Therefore, we began by studying a model in which the rigorous $\lambda$ POs of the layer group $P(c) c 2$ were represented by binary random variables that stand in, respectively, for the black or white chains in Fig. 12. Each chain is associated with such a variable, $\sigma$, which can take a value of either 0 for a black chain or 1 for a white chain. Different degrees of short-range order can be introduced into this system of random variables (and hence the corresponding molecules) with a Monte Carlo simulation using an energy of the form:

$$
\begin{aligned}
E= & \sum_{i=1}^{64} \sum_{j=1}^{64} \sum_{k=1}^{64}\left(J_{1}^{a} \sigma_{i j k} \sigma_{i \pm 1 j k}+J_{2}^{a} \sigma_{i j k} \sigma_{i \pm 2 j k}\right. \\
& \left.+J_{1}^{b} \sigma_{i j k} \sigma_{i j \pm 1 k}+J_{1}^{c} \sigma_{i j k} \sigma_{i j k \pm 1}\right) .
\end{aligned}
$$

Here $J_{1}^{a}, J_{2}^{a}$ etc. are effective interactions that may be adjusted in order to obtain desired values of the correlations, $C_{i}$, between neighbouring molecules in different crystal directions. We used the interactions between first-nearest-neighbour chains along the $c$ and $b$ axes and first- and secondnearest-neighbour chains along the $a$ direction. The MC calculation used an array of $64 \times 64 \times 64 \sigma_{i j k}$ variables, which correspond to $32 \times 32 \times 64$ unit cells of the structure, and each unit cell contains four independent chains. Overall the simulation involved 262144 chains and 6029312 atoms. Diffraction patterns were computed using the program DIFFUSE (Butler \& Welberry, 1992). This obtains the calculated diffuse diffraction pattern by averaging the diffraction patterns obtained from a large number of subregions (lots) taken at random from the total simulation array. In the present case, 50 individual lots of $4 \times 4 \times 15$ unit cells were used. Calculations were made on the $200 \times 200$ grid used for the observed patterns. The total CPU time for a single calculated pattern with these specifications was $\sim 15$ min on a DEC DS20 


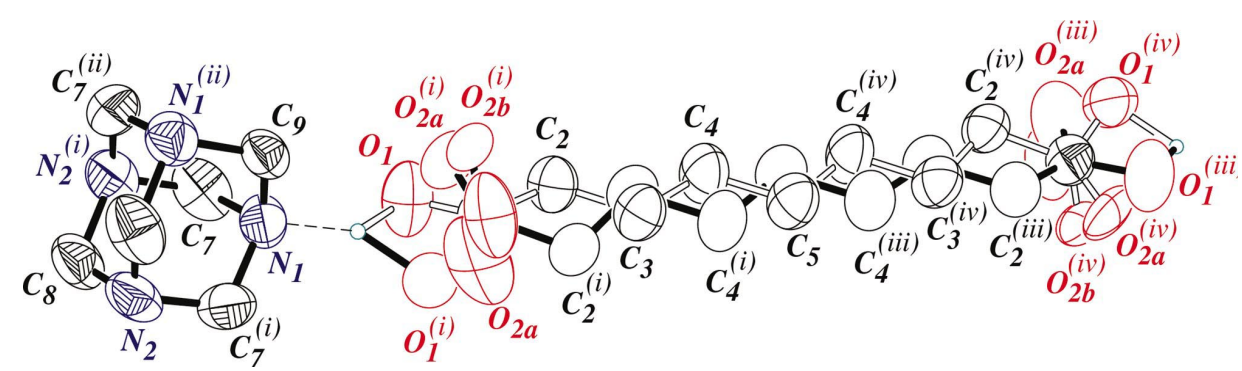

Figure 12

The two disordered chains in UA. The chain with the full bonds will be referred to as the black chain, the chain with the open bonds as the white chain. ${ }^{(i)}$ stands for $\sigma_{x} \mid 100,{ }^{(i i)}$ for $\sigma_{y} \mid 0 \frac{1}{2} 0,{ }^{(i i i)}$ for $C_{i} \mid 11 \overline{1},{ }^{(i v)}$ for $C_{2} x \mid 01 \overline{1}$. Atoms are drawn at the $50 \%$ probability level and methylene $\mathrm{H}$ atoms are omitted for the sake of clarity.

processor. Using correlations $C_{1}^{a} \simeq 0.6, C_{2}^{a} \simeq 0.2, C_{1}^{b} \simeq-0.7$ and $C_{1}^{c} \simeq-0.7$ we obtain a satisfactory correspondence for the $(\xi k \ell)$ layers with $k$ odd (Figs. $6 a$ and $6 c$ ) but disappointingly disparate relative intensities for the diffuse rods in the layers with $k$ even (not shown). The relative intensities for the $(\xi 2 \ell)$ rods were very-weak-strong-strong-weak-strong for $\ell=1,3,5,7,9$, whereas the observed values (Fig. $6 b$ ) were medium-strong-weak-very-weak for $\ell=1,3,5,7$. Since the correlations only affect the shape of the diffuse peaks and their intensity is related to the difference of the structure factors of the two types of molecule, it was clear that we needed to modify the structure of the layers in order to obtain a better fit. Consequently we tried modifying the molecules in various ways: for example, rotating the carboxyl groups at one chain end in every second layer, shifting the whole or parts of every second layer in all reasonable directions, and rotating the whole chains. None of these modifications resulted in any significant improvement. At this point, however, we obtained results for phase II of urotropin pimalate (UP) (Gardon, Pinheiro \& Chapuis, 2003) and this suggested a possible solution. In Fig. 13 we show the $b c$ projections of UA and UP. The structure of UA certainly justifies our first decision to include only the chains in our MC model. Although $a$ posteriori one might suspect some degree of rotational disorder around the $C_{2 z}$ axis, we had decided that the $\mathrm{U}$ molecules did not contribute to the diffuse scattering. However, in UP the same U molecules are clearly turned around the $\mathbf{c}$ axis by $\sim 10^{\circ}$, and every second layer is different too. Consequently we decided to include the $\mathrm{U}$ molecules of UP in our MC model for UA and obtained the $(\xi 4 \ell)$ layer shown in Fig. $6(d)$. The agreement with the experimental pattern is now qualitatively much more satisfactory: i.e. the profiles along the rods, e.g. ( $\xi 17)$, display a qualitative (number and positions of maxima) correspondence with the profiles observed in the $Q$-scans. By varying the $C_{1}^{a}$ and $C_{2}^{a}$ correlations it was even possible to toggle between the two MDO structures and their intermediate OD states, but it was considered too time-consuming to adjust these correlations by trial and error in order to obtain a quantitative fit of intensities. Also note that the image reconstructions (Figs. $6 a$ and $6 b$ ) were of relatively poor quality compared with the data obtainable from the Weißenberg PSD diffractometer system (Figs. $7 a$ and $7 b$ ) and were far too noisy to allow more than a qualitative fit. A more detailed analysis is underway, which uses a more sophisticated MC automatic refinement technique (Welberry et al., 1998) that uses $Z$-matrices. A preliminary report of this analysis has been presented elsewhere (Goossens \& Welberry, 2001).

The $(1 k \ell)$ and $(2 k \ell)$ sections of data shown in Figs. 7(a) and 7(b) contain diffuse peaks that correspond to the cross-section of the diffuse rods that are seen in Figs. $6(a)$ and $6(b)$. In addition these layers show rather broad rhomb-shaped diffuse bands. In order to reproduce these bands in the simulated patterns each chain in our $32 \times 32 \times 64$ unit cells was shifted by a random displacement of between -0.5 and $+0.5 \AA$ along its chain axis. With this simple addition to the model described above the simulated layers shown in Figs. 7(c) and 7(d) were obtained. The ( $0 k \ell)$ layer (not shown) does not contain any SRO peaks but the rhomb-shaped diffuse bands were reproduced here too.

The observed and calculated $(1 k \ell)$ and $(2 k \ell)$ reciprocal layers displayed in Fig. 7 show generally broad agreement. The SRO peaks are seen to occur in the $\ell=$ odd reciprocal rows (vertical) and the distributions of peak intensities along these rows show qualitative similarity. Note that the strongest peaks occur in regions where the broad diffuse bands, which result from the chain displacements, are also strong. This simple relationship reflects the fact that for both the short-range order and the displacements the scattering comes predominantly from the azelaic acid molecules. In view of the simplistic nature of the present model this level of agreement can be considered satisfactory. Clearly the detailed distribu-
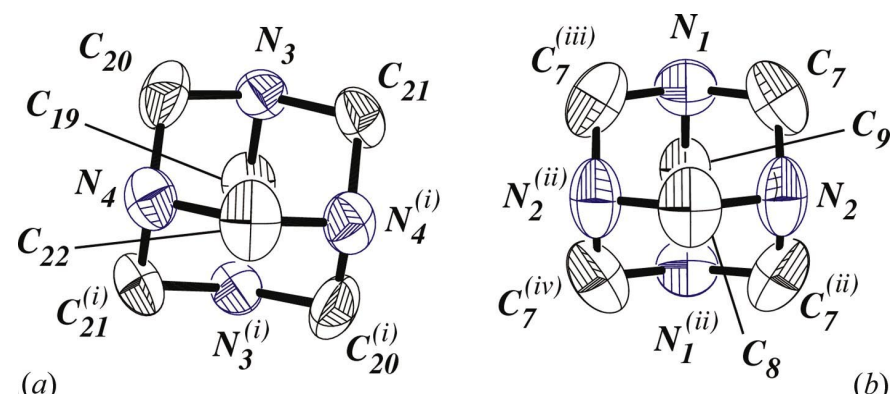

\section{Figure 13}

(a) The U molecule in a layer of UP has $C_{2}$ site symmetry. ${ }^{(i)}$ stands for $C_{2} z \mid 000,{ }^{(i i)}$ for $\sigma_{y} \mid 0 \frac{1}{2} 0,{ }^{(i i i)}$ for $\sigma_{x} \mid 100$ and ${ }^{(i v)}$ for $C_{2} z \mid 1 \frac{1}{2} 0$. This structure corresponds to the pure 'screwed' MDO. (b) The U molecule in a layer of UA has site symmetry $C_{2 v}$. The symmetry codes are the those used in Fig. 12. Atoms are drawn at the $50 \%$ level and $\mathrm{H}$ atoms are omitted for the sake of clarity. 
tion of intensity is very sensitive to the exact detail of the two molecular form factors $F_{\text {black }}, F_{\text {white }}$.

It is also noticeable that the peak maxima do not occur precisely on the $\ell$ integral positions but are shifted sideways to varying degrees. Again the overall pattern of these shifts is qualitatively reasonably well reproduced in the simulated patterns. In many systems such peak shifts occur as a result of size-effect distortions (Welberry \& Mayo, 1996), but in this case the shifts are found to occur when no such effects have been included in the model. The shifts occur because of the steep gradients in the overall diffuse intensity distribution, which is proportional to the difference in the two molecular form factors $\left|F_{\text {black }}-F_{\text {white }}\right|^{2}$.

Clearly the present model in which the molecules are shifted at random along the length of the chain is a very crude approximation. In practice we would expect such shifts to be correlated from molecule to molecule and to be cross-correlated with the occupational disorder (i.e. with $\sigma$ ). The observed patterns provide clear evidence of the former effect, which generally tends to produce diffuse scattering in the neighbourhood of strong Bragg peaks. In particular the strong elongated diffuse peaks that occur close to the (124) and (125) reflections can be attributed to such correlation of the molecular displacements.

It is also worth mentioning that if the displacement shifts along the chain axes are considered to be coupled to the occupational disorder then the effects that are seen in phase II may be considered as precursors of the modulations that are formed in UA at higher temperatures. For example, modulation vectors of the type $\alpha \mathbf{a}_{o}+\gamma \mathbf{c}_{o}$ with $\alpha \simeq-0.02$ and $\gamma \simeq 0.24$ have been found between $\sim 351$ and $\sim 363 \mathrm{~K}$ (Gardon, 2001).

\section{Conclusions}

The tale of $\mathrm{U}$ and AA is the story of a shaky marriage. The two components are able to form a co-crystal, but this is far from being a unified and homogeneous object. Each partner appears to take every opportunity to impose itself on the other or, failing this, to break apart from it. Our study has shown that it is possible to design a clearly novel system that exhibits unusual, even exotic, properties by mixing the appropriate components. The co-crystals are stable only under rather precise values of chemical potential, temperature or pressure and dissociate under even a slight deviation from these equilibrium conditions. Note, though, that if the co-crystals are kept under even a modest pressure, say $\sim 6 \times 10^{5} \mathrm{~Pa}$, they display a completely different behaviour with more classical, but less interesting, phase transitions and are stable up to the melting point (Bonin et al., 2003).

The association of AA and $\mathrm{U}$ in their mother liquor is very tenuous. It is probably easier to form aggregates of $U$ alone (for geometric reasons) or of AA alone (because of existing micelles) than aggregates of UA. For this reason we believe that the nucleation of UA initiates from existing seeds of the $\alpha$ form of AA and that these will partly survive in the UA cocrystal. The role of the small percentage of included aceto- nitrile might be to stabilize the grain boundaries between the AA and UA domains since these do not exhibit a very favourable epitaxic ratio. We also believe that there is always a certain amount of U alone at the (010) surfaces of a UA cocrystal.

The UA co-crystals are thus considered to be quite inhomogenous objects that contain a wide variety of defects including solvent inclusions, parasite phases, dislocations, point defects and surface rearrangements. It is difficult to conceive experiments that could yield precise information on all these defects since their total concentration must be less than a few percent. Nevertheless, the various experimental techniques described in this paper have provided evidence for the existence of these defects, and some of them clearly play a crucial role in the phase transitions at higher temperatures (Gardon, Schenk et al., 2003).

The percentage of the AA domains [their existence is proven by the powder rings in Figs. 7(a) and 7(b)] will vary from crystal to crystal, as will the concentration of acetonitrile (shown by GC) and the precise stacking sequence of the (100) layers (echoed in the rods of diffuse scattering), but all of these effects are necessary to account for the often surprising phenomena in UA. For example, the exotic phase front phenomena described earlier seem to depend on the size of the crystal studied; this behaviour now becomes understandable in the light of our picture of the inhomogenous nature of the crystals.

The acetonitrile inclusions play a crucial role in the surface cleansing at $\sim 338 \mathrm{~K}$ and in the recrystallization of $\mathrm{U}$ on the (010) surface (Gardon, Schenk et al., 2003). The AA regions within the co-crystal might furnish an even better model for the $\beta \rightarrow \gamma$ transition that is accompanied by the spectacular phase fronts at $\sim 351 \mathrm{~K}$. (The similarity of this temperature to that of the $\beta \rightarrow \alpha$ transition in pure AA may be significant.) The acetonitriles and $U$ are again very probably essential for the diffusion-controlled mechanisms at low temperature.

The packing of UA is less compact than that of either single component. Consequently, UA is characterized by a greater amount and a more diverse range of molecular motions and more defects than either AA or especially U. In that sense it may be said that UA is different from, rather than similar to, either AA or U. While it is true that certain properties of the original components persist in the co-crystal, they are amalgamated to lend UA a much richer spectrum of features. For example, the (110) $)_{c}$ layer of $U$ is preserved in the co-crystal with only very minor changes and similarly the instabilities due to the tendency of $\mathrm{U}$ to sublime are also preserved. AA provides, via the carboxyl group, the major and some of the minor hydrogen bonds in UA. The inclination of AA to form polymorphs probably contributes to the wealth of phase transitions of UA and its deprotonation ambiguity is preserved as well [phases II and III (Hostettler et al., 1999)]. However, some features such as the number and variety of phase transitions are clearly specific to UA and are due to the exceptional inhomogeneity and the very complex real structure. For example, the profusion of hydrogen bonding and the rather uncommon dynamics (e.g. non-localized vibrations) of the AA 
chains that are strung between pairs of $U$ molecular layers are not present in crystals of the individual components.

In order to explain the spectacular phase fronts that have been seen in the study of the $\beta \rightarrow \gamma$ transition (Gardon, Schenk et al., 2003) in UA, a novel diffusion-controlled competition between two slightly different incommensurate phases is postulated.

In phase $\alpha$ at, say, $T \simeq 303 \mathrm{~K}$, the homologous temperature, $T_{h} \simeq 0.75$, is indicative of a 'recrystallization' regime. Indeed, this regime extends over the whole temperature range within which the phase transitions occur. The system is therefore characterized by high diffusion (of vacancies, $U$ molecules and acetonitriles) and important thermal fluctuations. These entail movement of dislocations, domain walls and grain boundaries. Nevertheless, the average structure is well defined. The stacking of the (100) layers is quasi-random (Fig. 11). The weak interlayer forces together with the internal stress at the various boundaries $(U A \leftrightarrow A A, U A \leftrightarrow U)$ and at the diverse point defects create the prerequisites for the thermal activation of edge dislocations with a Burgers vector $\mathbf{c} / 4$. With changing temperature the stacking sequence can therefore change as well.

At $T_{\alpha \rightarrow \text { II }}$ the building-up of the screwed MDO sets in. We believe (although this hypothesis will have to be subjected to the experimental test of vibrational and NMR spectroscopies) that this build-up is triggered by the locking-in of the four-site hopping of the $\mathrm{U}$ molecules. Once they are locked, they can establish $\mathrm{C}-\mathrm{H} \cdots \mathrm{N}$ hydrogen bonds with neighbouring $\mathrm{U}$ molecules. This interaction, though weak, is structurally important. (We note that in $\mathrm{U}$ this interaction prevents the crystal from being a plastic crystal like adamantane.) These bonds are along [101]/[101] and the progression of their establishment provides a mechanistic explanation for the $(101) /(10 \overline{1})$ phase fronts. The formation of these bonds results in the creation of a two-dimensional network. Concomitantly, this advancement provides the internal stress necessary for dislocation movement favourable to the creation of the screwed MDO. Note that this latter is compatible with the orthorhombic metric of the OD structure and does not require a spontaneous macroscopic deformation.

Between $293 \mathrm{~K}$ and $T_{\mathrm{II} \rightarrow \mathrm{III}}$ the domains of the screwed MDO grow thicker. It appears that the MDO would eventually be converted to a pure three-dimensional structure with space group Pcc2 (like UP; Gardon, Pinheiro \& Chapuis, 2003 ), although at a lower temperature. At $\sim 283 \mathrm{~K}$ similar transitions occur in UP and UA: in the former from a threedimensional 'screwed' MDO to a three-dimensional 'slated' $\mathrm{MDO}$, and in the latter from a mixture of 'screwed' and 'slated' MDOs to a three-dimensional 'slated' MDO. Since such similar phenomena appear at such similar temperatures in the two materials, the initiator of the transition ought to be a common factor present in both compounds. This hypothesis is certainly true for the $U$ (010) layer, and therefore we conjecture that a soft mode that involves the $\mathrm{U}$ molecules of such a layer starts the II $\rightarrow$ III transition. However, the transition does not proceed in the same manner in the two compounds. Phase II of UP may be understood as a regular stacking of microscopic (i.e. just two layers thick) ferroelastic (100) domains of phase III (Fig. 14a). All UP would have to do during the transition is to increase the size of these domains (in UA they consist of $\sim 200$ layers), and indeed this layer rearrangement can be achieved by means of an enthalpy of $\sim 1 \mathrm{~J} \mathrm{~g}^{-1}$. In UA the layer stacking is less regular and some regions are already in the 'right' sequence, but strangely enough the presence of the 'slated' regions does not seem to help the transition. Possibly, this behaviour occurs because the process of growing larger domains from the 'slated' nuclei that are already present might be costly in terms of elastic energy. In any case the direct II $\rightarrow$ III transition is not possible and the system first grows a furtive triggering phase from which it can then build up the 'slated' stacking more easily $\left(\Delta H \simeq 2 \mathrm{~J} \mathrm{~g}^{-1}\right)$. The intermediate phase, $f$, is likely to be triclinic since the $40 \overline{1}$ domains do not correspond to any symmetry direction of the orthorhombic parent phase. After the condensation of the soft mode, the hydrogen bonds on one side of the $\sigma_{x}$ mirror plane become stronger (shorter by roughly $0.1 \AA$ ). This preference leads then to the growth of phase III. On a microscopic level the transition involves the propagation (much like falling dominos) of these shorter hydrogen bonds along [101]/[101] . As a consequence of this bias and of the symmetry compatibility (monoclinic metric) the slated MDO (space group $P 2_{1} / c$ ) then becomes the more stable and the (100) layers are shifted by dislocation movement to build up the structure. Again, the decisive reconstruction takes place along the diagonals of the orthorhombic cell and this explains the $(101) /(10 \overline{1})$ phase fronts.

It is thus seen that the phase sequences in UA and UP are really quite similar; the major difference lies in the conversion of the screwed MDO to a fully fledged three-dimensional structure in UP, whereas in UA this conversion is by-passed by the condensation of the soft mode, which leads to the nucleation and growth of phase III. In the $\alpha$ phase the screwed MDO is being formed. When this is converted to the perfect

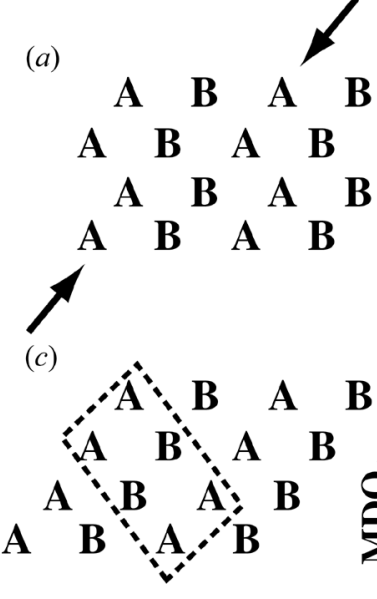

(b)
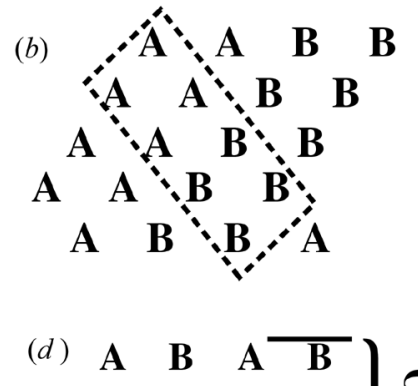

Figure 14

Chain and layer sequence in $(a) \mathrm{MDO}_{1},(b)$ phase IV, (c) phase III, $\mathrm{MDO}_{2}$. (d) Stacking fault leading to an $\mathrm{MDO}_{1}$ domain wall. 
three-dimensional structure, considerable energy is released [in the case of UP (Gardon, Pinheiro \& Chapuis, 2003) $\Delta H \sim 17.3 \mathrm{~J} \mathrm{~g}^{-1}$. In the case of UA the condensation of the soft mode occurs before this conversion and the energy is kept within the system. Phase II is therefore a more energetic state. This excess energy can then be used to activate the $\left\{(\overline{1} 51) \mid \mathbf{t}=\mathbf{a}_{m}\right\}$ slip system during the III $\rightarrow$ IV transition.

In the screwed MDO there exists an $A A B B$ sequence (Fig. 14a) of chains along [101]/[101] . The sequence in the neighbouring row is the same, but shifted by $\mathbf{a}_{m}$. The same chain sequence also exists in phase IV, and the system is thus very close to its ground state. (Incidentally, this similarity to the ground state also means that phase IV cannot be described as emerging from the OD structure.) All the system had to do was to shift alternate rows by opposite displacements. Obviously, this process is energetically too expensive in UP and UA, but such shifts do occur in urotropin undecanoate [UU] (Pinheiro et al., 2003). There is no intermediate phase and the system goes directly from the screwed MDO to phase IV (Fig. 14b).

In UA the activation of this slip system has to wait until $T \simeq 233 \mathrm{~K}$, at which temperature the transition takes place. This transformation is achieved by a combination of two slip systems: the $\{(100) \mid \mathbf{t}=\mathbf{c} / 4\}$ already used above and the more energetic $\left\{(\overline{1} 51) \mid \mathbf{t}=\mathbf{a}_{m} / 4\right\}$. The latter and its equivalents produce displacements of [101]/[101] rows of chains accompanied by displacements along (100). Nucleation of phase IV may involve an occasional residual domain of screwed MDO (Fig. 14d). Again, the reconstruction proceeds along the (101)/(101) phase fronts. For the $\left\{(\overline{151}) \mid \mathbf{t}=\mathbf{a}_{m}\right\}$ slip system to cross the ubiquitous $\{(100) \mid \mathbf{t}=\mathbf{c} / 4\}$ system is difficult and can lead to climb, cross slip and sessile dislocations. As a result the crystal may be torn apart; its fate depends on its precise microstructure. In this context we should mention that a synchrotron powder diffraction experiment has shown that the already thin (100) domains in phase III are reduced to really tiny regions (along $\mathbf{a}$ and $\mathbf{c}$ ). These lead to considerable line broadening in phase IV.

Let us finally mention (Mucciolò, 1999) that these ubiquitous $(101) /(10 \overline{1})$ phase fronts have also been observed in UP.

We sincerely thank Dr Monique Vogel for elucidating discussions on the biological aspects of AA. We express our heartfelt gratitude towards Antonio Mucciolò for his competent and generous help with the orthoscopic experiments. HB gratefully acknowledges the Danish Research Training Council for financial support.

\section{References}

Abrahamsson, S., Dahlén, B., Löfgren, H. \& Pascher, I. (1978). Prog. Chem. Fats Other Lipids, 16, 125-143.
Birkedal, H. (1996). Diploma Thesis, Copenhagen University.

Birkedal, H., Gardon, M., Hostettler, M., Duc, F., Schwarzenbach, D., Chapuis, G. \& Bonin, M. (2003). In preparation.

Blažević, N., Kolbah, D., Belin, B., Šunjič, V. \& Kajfež, F. (1979). Synthesis, pp. 161-176.

Bloss, F. D. (1990). An Introduction to the Methods of Optical Crystallography, ch. VIII. Marietta, OH 454750, USA: RAN Publishers. [Interference colour chart.]

Bond, A. D., Edwards, M. R. \& Jones, W. (2001). Acta Cryst. E57, o143-o144.

Bonin, M., Bonin, L. G., Ĉerny, R. \& Schenk, K. J. (2003). In preparation.

Bonin, M., Fuith, A., Schenk, K. J. \& Tolédano, P. (1999). Phys. Rev. 59, 14246-14252.

Butler, B. D. \& Welberry, T. R. (1992). J. Appl. Cryst., 25, 391-399.

Canut, M. L. \& Amorós, J. L. (1958). Bol. Real Soc. Espan. Hist. Nat. G56, 323-338.

Caspari, W. A. (1929). J. Chem. Soc. pp. 2709-2712.

DiPersio, J. \& Escaig, B. (1972). Crystal Lattice Defects, 3, 55-58.

Dornberger-Schiff, K. (1966). Lehrgang über OD-Strukturen. Akademie Verlag Berlin.

Dupré La Tour, F. (1935). C. R. Acad. Sci. 201, 479-481.

Gardon, M. (2001). PhD thesis, University of Lausanne, Switzerland.

Gardon, M., Pinheiro, C. B. \& Chapuis, G. (2003). In preparation.

Gardon, M., Schenk, K. J., Bonin, M. \& Tolédano, P. (2003). In preparation.

Geday, M. A. \& Glazer, A. M. (2002). J. Appl. Cryst. 35, 185-190.

Goossens, D. J. \& Welberry, T. R. (2001). Comput. Phys. Commun. 142, 387-390.

Guinier, A. (1964). Théorie et technique de la radiocristallographie, ch. XIII, 3rd ed. Paris: Dunod.

Harburn, G., Taylor, C. A. \& Welberry, T. R. (1975). Atlas of Optical Transforms, Plate 24. London: G. Bell and Sons.

Holakovský, J. (1973). Phys. Status Solidi B, 56, 615-619.

Hostettler, M. Birkedal, H., Gardon, M., Chapuis, G., Schwarzenbach, D. \& Bonin, M. (1999). Acta Cryst. B55, 448-458.

Housty, J. \& Hospital, M. (1967). Acta Cryst. 22, 288-295.

Jeffrey, G. A. \& Sänger, W. (1991). Hydrogen Bonding in Biological Structures. Berlin: Springer Verlag.

Kampermann, S. P., Sabine, T. M., Craven, B. M. \& McMullan, R. K. (1995). Acta Cryst. A51, 489-497.

Klipping, G. \& Stranski, I. N. (1958). Z. Anorg. Allg. Chem. 297, $23-$ 31.

Mucciolò, A. (1999). Private communication.

Osborn, J. C. \& Welberry, T. R. (1990). J. Appl. Cryst. 23, 476-484.

Pinheiro, C. B., Gardon, M. \& Chapuis, G. (2003). In preparation.

Salem, L. (1962). J. Chem. Phys. 37, 2100-2113.

Schenk, K. J. (1984). PhD thesis, University of Lausanne, Switzerland.

Sheldrick, G. M. (1997a). SHELXL97. Program for the Refinement of Crystal Structures. University of Göttingen, Germany.

Sheldrick, G. M. (1997b). SHELXS97. Program for the Solution of Crystal Structures. University of Göttingen, Germany.

Stamatiadis, D., Bulteau-Portois, M. C. \& Mowszowicz, I. (1988). Br. J. Dermatol. 119, 627-632.

Steiner, T. \& Desiraju, G. R. (1998). Chem. Commun. pp. 891-892.

Stoe \& CIE GmbH (1997). IPDS 2.89 Software Manual. Stoe and CIE, Darmstadt, Germany.

Thalladi, V. R., Nüsse, M. \& Boese, R. (2000). J. Am. Chem. Soc. 122, 9227-9236.

Welberry, T. R. \& Mayo, S. C. (1996). J. Appl. Cryst. 29, 353-364.

Welberry, T. R., Proffen, Th. \& Bown, M. (1998). Acta Cryst. A54, 661-674. 